\title{
UNSOLID AND FLUID STRONG VARIETIES OF PARTIAL ALGEBRAS
}

\author{
S. BUSAMAN AND K. DENECKE
}

Received 8 January 2006; Revised 14 August 2006; Accepted 21 August 2006

A partial algebra $\mathscr{A}=\left(A ;\left(f_{i}^{A}\right)_{i \in I}\right)$ consists of a set $A$ and an indexed set $\left(f_{i}^{A}\right)_{i \in I}$ of partial operations $f_{i}^{A}: A^{n_{i}} \multimap \rightarrow A$. Partial operations occur in the algebraic description of partial recursive functions and Turing machines. A pair of terms $p \approx q$ over the partial algebra $A$ is said to be a strong identity in $\mathscr{A}$ if the right-hand side is defined whenever the left-hand side is defined and vice versa, and both are equal. A strong identity $p \approx q$ is called a strong hyperidentity if when the operation symbols occurring in $p$ and $q$ are replaced by terms of the same arity, the identity which arises is satisfied as a strong identity. If every strong identity in a strong variety of partial algebras is satisfied as a strong hyperidentity, the strong variety is called solid. In this paper, we consider the other extreme, the case when the set of all strong identities of a strong variety of partial algebras is invariant only under the identical replacement of operation symbols by terms. This leads to the concepts of unsolid and fluid varieties and some generalizations.

Copyright (c) 2006 Hindawi Publishing Corporation. All rights reserved.

\section{Introduction}

Let $P^{n}(A):=\left\{f: A^{n} \multimap \rightarrow A\right\}$ be the set of all $n$-ary partial operations defined on the set $A$ and let $P(A):=\bigcup_{n=1}^{\infty} P^{n}(A)$ be the set of all partial operations on $A$. A partial algebra $\mathscr{A}=\left(A ;\left(f_{i}^{A}\right)_{i \in I}\right)$ of type $\tau=\left(n_{i}\right)_{i \in I}$ is a pair consisting of a set $A$ and an indexed set $\left(f_{i}^{A}\right)_{i \in I}$ of partial operations where $f_{i}^{A}$ is $n_{i}$-ary. Let $P \operatorname{Alg}(\tau)$ be the class of all partial algebras of type $\tau$. Let $X_{n}:=\left\{x_{1}, \ldots, x_{n}\right\}$ be a finite alphabet and let $X:=\left\{x_{1}, \ldots, x_{n}, \ldots\right\}$ be a countable infinite alphabet. Let $W_{\tau}\left(X_{n}\right)$ be the set of all $n$-ary terms of type $\tau$ and let $W_{\tau}\left(X_{n}\right)^{\mathscr{A}}$ be the set of all $n$-ary induced term operations on the partial algebra $\mathscr{A}$ (see, e.g., [3] ). Since in general the set $W_{\tau}\left(X_{n}\right)^{\mathscr{A}}$ is different from the set of all partial operations generated by $\left\{f_{i}^{\mathcal{A}} \mid i \in I\right\}$ we need a new definition of terms over partial algebras of type $\tau$.

Let $\left\{f_{i} \mid i \in I\right\}$ be a set of operation symbols of type $\tau$, where each $f_{i}$ has an arity $n_{i}$ and $X \cap\left\{f_{i} \mid i \in I\right\}=\varnothing$. We need additional symbols $\varepsilon_{j}^{k} \notin X$, for every $k \in \mathbb{N}^{+}:=\mathbb{N} \backslash\{0\}$ and $1 \leq j \leq k$. The set of $n$-ary $C$-terms of type $\tau$ over $X_{n}$, for short we will speak of $n$-ary terms, is defined inductively as follows (see [1]). 
2 Unsolid and fluid strong varieties of partial algebras

(i) Every $x_{i} \in X_{n}$ is an $n$-ary term of type $\tau$.

(ii) If $w_{1}, \ldots, w_{k}$ are $n$-ary terms of type $\tau$, then $\varepsilon_{j}^{k}\left(w_{1}, \ldots, w_{k}\right)$ is an $n$-ary term of type $\tau$ for all $1 \leq j \leq k$ and all $k \in \mathbb{N}^{+}$.

(iii) If $w_{1}, \ldots, w_{n}$ are $n$-ary terms of type $\tau$ and if $f_{i}$ is an $n_{i}$-ary operation symbol, then $f_{i}\left(w_{1}, \ldots, w_{n_{i}}\right)$ is an $n$-ary term of type $\tau$.

Let $W_{\tau}^{C}\left(X_{n}\right)$ be the set of all $n$-ary terms of type $\tau$ defined in this way. Then $W_{\tau}^{C}(X):=$ $\bigcup_{n=1}^{\infty} W_{\tau}^{C}\left(X_{n}\right)$ denotes the set of all terms of this type. Note that here the use of the superscript $C$ will distinguish these sets from the analogous ones in the total case. The letter $C$ was used since Craig $[4,5]$ suggested the addition of the extra constant term $\varepsilon_{j}^{k}$. The main reason to introduce $C$-terms is connected with induced term operations which we want to define now.

Every $n$-ary term $w \in W_{\tau}^{C}\left(X_{n}\right)$ induces an $n$-ary term operation $w^{A}$ on any partial algebra $\mathscr{A}=\left(A ;\left(f_{i}^{\mathscr{A}}\right)_{i \in I}\right)$ of type $\tau$. For $a_{1}, \ldots, a_{n} \in A$, the value $w^{\mathscr{A}}\left(a_{1}, \ldots, a_{n}\right)$ is defined in the following inductive way (see [1]).

(i) If $w=x_{i}$, then $w^{\mathscr{A}}=x_{i}^{A}=e_{i}^{n, A}$, where $e_{i}^{n, A}$ is as usual the $n$-ary total projection on the $i$ th component.

(ii) If $w=\varepsilon_{j}^{k}\left(w_{1}, \ldots, w_{k}\right)$ and we assume that $w_{1}^{A}, \ldots, w_{k}^{\mathscr{A}}$ are the term operations induced by the terms $w_{1}, \ldots, w_{k}$ and that the $w_{i}^{\mathscr{A}}\left(a_{1}, \ldots, a_{n}\right)$ are defined for $1 \leq i \leq k$, then $w^{\mathscr{A}}\left(a_{1}, \ldots, a_{n}\right)$ is defined and $w^{\mathscr{A}}\left(a_{1}, \ldots, a_{n}\right)=w_{j}^{\mathscr{A}}\left(a_{1}, \ldots, a_{n}\right)$.

(iii) Now assume that $w=f_{i}\left(w_{1}, \ldots, w_{n_{i}}\right)$ where $f_{i}$ is an $n_{i}$-ary operation symbol, and assume that the $w_{j}^{A A}\left(a_{1}, \ldots, a_{n}\right)$ are defined, with values $w_{j}^{A A}\left(a_{1}, \ldots, a_{n}\right)=b_{j}$ for $1 \leq$ $j \leq n_{i}$. If $f_{i}^{A}\left(b_{1}, \ldots, b_{n_{i}}\right)$ is defined, then $w^{A}\left(a_{1}, \ldots, a_{n}\right)$ is defined and $w^{A}\left(a_{1}, \ldots\right.$, $\left.a_{n}\right)=f_{i}^{A}\left(w_{1}^{A}\left(a_{1}, \ldots, a_{n}\right), \ldots, w_{n_{i}}^{A A}\left(a_{1}, \ldots, a_{n}\right)\right)$.

On the sets $W_{\tau}^{C}\left(X_{n}\right)$ we may introduce the following superposition operations. Let $w_{1}, \ldots, w_{m}$ be $n$-ary terms and let $t$ be an $m$-ary term. Then we define an $n$-ary term $\bar{S}_{n}^{m}\left(t, w_{1}, \ldots, w_{m}\right)$ inductively by the following steps.

(i) For $t=x_{j}, 1 \leq j \leq m$ ( $m$-ary variable), we define

$$
\bar{S}_{n}^{m}\left(x_{j}, w_{1}, \ldots, w_{m}\right)=w_{j} .
$$

(ii) For $t=\varepsilon_{j}^{k}\left(s_{1}, \ldots, s_{k}\right)$ we set

$$
\bar{S}_{n}^{m}\left(t, w_{1}, \ldots, w_{m}\right)=\varepsilon_{j}^{k}\left(\bar{S}_{n}^{m}\left(s_{1}, w_{1}, \ldots, w_{m}\right), \ldots, \bar{S}_{n}^{m}\left(s_{k}, w_{1}, \ldots, w_{m}\right)\right),
$$

where $s_{1}, \ldots, s_{k}$ are $m$-ary terms, for all $k \in \mathbb{N}^{+}$and $1 \leq j \leq k$.

(iii) For $t=f_{i}\left(s_{1}, \ldots, s_{n_{i}}\right)$ we set

$$
\bar{S}_{n}^{m}\left(t, w_{1}, \ldots, w_{m}\right)=f_{i}\left(\bar{S}_{n}^{m}\left(s_{1}, w_{1}, \ldots, w_{m}\right), \ldots, \bar{S}_{n}^{m}\left(s_{n_{i}}, w_{1}, \ldots, w_{m}\right)\right),
$$

where $s_{1}, \ldots, s_{n_{i}}$ are $m$-ary terms.

This defines an operation

$$
\bar{S}_{n}^{m}: W_{\tau}^{C}\left(X_{m}\right) \times\left(W_{\tau}^{C}\left(X_{n}\right)\right)^{m} \longrightarrow W_{\tau}^{C}\left(X_{n}\right),
$$

which describes the superposition of terms. 
The term clone of type $\tau$ is the heterogeneous algebra

$$
\text { clone } \tau^{C}:=\left(W_{\tau}^{C}\left(X_{n}\right) ; \bar{S}_{n}^{m}, e_{j}^{k}\right)_{n, m, k \in \mathbb{N}^{+}, 1 \leq j \leq k},
$$

where $e_{j}^{k}:=x_{j} \in X_{k}, 1 \leq j \leq k$.

(Since in this paper we will only use $C$-terms, we will speak of the term clone of type $\tau$ instead of the $C$-term clone of type $\tau$.)

Superposition can be defined for partial operations on $A$, as it was done in $[8$, Section 4.5] for total operations. We define operations $S_{n}^{m}: P^{m}(A) \times\left(P^{n}(A)\right)^{m} \rightarrow P^{n}(A)$ by

$$
S_{n}^{m}\left(f^{A}, g_{1}^{A}, \ldots, g_{m}^{A}\right)\left(a_{1}, \ldots, a_{n}\right):=f^{A}\left(g_{1}^{A}\left(a_{1}, \ldots, a_{n}\right), \ldots, g_{m}^{A}\left(a_{1}, \ldots, a_{n}\right)\right),
$$

for all $\left(a_{1}, \ldots, a_{n}\right)$ for which $g_{1}^{A}, \ldots, g_{m}^{A}$ are defined and for which the values $b_{1}=g_{1}^{A}\left(a_{1}, \ldots\right.$, $\left.a_{n}\right), \ldots, b_{m}=g_{m}^{A}\left(a_{1}, \ldots, a_{n}\right)$ form an $m$-tuple $\left(b_{1}, \ldots, b_{m}\right)$ belonging to the domain of $f^{A}$.

Any subalgebra of the heterogeneous algebra

$$
\left(P^{n}(A) ; S_{n}^{m}, e_{i}^{n, A}\right)_{n, m \in \mathbb{N}^{+}, 1 \leq i \leq n}
$$

is called a partial clone on $A$.

We note that only the use of $C$-terms guarantees that the set of all term operations over the partial algebra $\mathscr{A}=\left(A ;\left(f_{i}^{A}\right)_{i \in I}\right)$ is equal to the partial clone generated by the set $\left\{f_{i}^{A} \mid i \in I\right\}$ of fundamental operations of $\mathscr{A}$.

\section{Regular hypersubstitutions and $M$-solid strong varieties}

In this section we recall some basis facts about regular hypersubstitutions, strong hyperidentities and solid varieties of partial algebras. For more details see $[3,6,7]$.

Definition 2.1 (see [11]). Let $\left\{f_{i} \mid i \in I\right\}$ be a set of operation symbols of type $\tau$ and let $W_{\tau}^{C}(X)$ be the set of all terms of this type. A mapping $\sigma:\left\{f_{i} \mid i \in I\right\} \rightarrow W_{\tau}^{C}(X)$ which maps each $n_{i}$-ary fundamental operation $f_{i}$ to a term of arity $n_{i}$ is called a hypersubstitution of type $\tau$. (Again one speaks of hypersubstitution of type $\tau$ instead of $C$-hypersubstitution of type $\tau$.)

Any hypersubstitution $\sigma$ of type $\tau$ can be extended to a map $\hat{\sigma}: W_{\tau}^{C}(X) \rightarrow W_{\tau}^{C}(X)$ defined for all terms, in the following way:

(i) $\hat{\sigma}\left[x_{i}\right]=x_{i}$ for every $x_{i} \in X_{n}$,

(ii) $\hat{\sigma}\left[\varepsilon_{j}^{k}\left(s_{1}, \ldots, s_{k}\right)\right]=\bar{S}_{n}^{k}\left(\varepsilon_{j}^{k}\left(x_{1}, \ldots, x_{k}\right), \hat{\sigma}\left[s_{1}\right], \ldots, \hat{\sigma}\left[s_{k}\right]\right)$, where $s_{1}, \ldots, s_{k} \in W_{\tau}^{C}\left(X_{n}\right)$,

(iii) $\hat{\sigma}\left[f_{i}\left(t_{1}, \ldots, t_{n_{i}}\right)\right]=\bar{S}_{n}^{n_{i}}\left(\sigma\left(f_{i}\right), \hat{\sigma}\left[t_{1}\right], \ldots, \hat{\sigma}\left[t_{n_{i}}\right]\right)$, where $t_{1}, \ldots, t_{n_{i}} \in W_{\tau}^{C}\left(X_{n}\right)$.

As Welke proved in [11], a necessary condition for $\hat{\sigma}[s] \approx \hat{\sigma}[t]$ to be a strong identity in a partial algebra $\mathscr{A}$ whenever $s \approx t$ is a strong identity in $\mathscr{A}$ is that $\sigma$ is regular.

So to define strong hyperidentities we will consider only regular hypersubstitutions.

Let $\operatorname{Var}(t)$ be the set of all variables occurring in the term $t$.

Definition 2.2 (see [9]). The hypersubstitution $\sigma$ is called regular if $\operatorname{Var}\left(\sigma\left(f_{i}\right)\right)=\left\{x_{1}, \ldots\right.$, $\left.x_{n_{i}}\right\}$, for all $i \in I$. 
4 Unsolid and fluid strong varieties of partial algebras

Let $\operatorname{Hyp}_{R}^{C}(\tau)$ be the set of all regular hypersubstitutions of type $\tau$.

Lemma 2.3 (see [11]). Let $\sigma_{1}, \sigma_{2} \in \operatorname{Hyp}_{R}^{C}(\tau)$. Then $\left(\hat{\sigma}_{2} \circ \sigma_{1}\right)^{\wedge}=\hat{\sigma}_{2} \circ \hat{\sigma}_{1}$, where $\circ$ is the usual composition of functions.

Now we define a product of hypersubstitutions in the usual way by $\sigma_{1}{ }^{\circ} \sigma_{2}:=\hat{\sigma}_{1} \circ \sigma_{2}$ and obtain the following.

Theorem 2.4 (see [11]). The algebra $\left(\operatorname{Hyp}_{R}^{C}(\tau) ;{ }^{\circ}, \sigma_{\text {id }}\right)$ with $\sigma_{\text {id }}\left(f_{i}\right)=f_{i}\left(x_{1}, \ldots, x_{n_{i}}\right)$ is a monoid.

Let $\mathscr{A}=\left(A ;\left(f_{i}^{\mathscr{A}}\right)_{i \in I}\right)$ be a partial algebra of type $\tau=\left(n_{i}\right)_{i \in I}$, and let $\sigma \in \operatorname{Hyp}_{R}^{C}(\tau)$. We want to consider the derived algebra $\sigma(\mathscr{A})=\left(A ;\left(\sigma\left(f_{i}\right)^{\mathscr{A}}\right)_{i \in I}\right)$, where $\sigma\left(f_{i}\right)^{\mathscr{A}}$ is the term operation induced by the term $\sigma\left(f_{i}\right)$ on the algebra $\mathscr{A}$.

Lemma 2.5 (see [11]). Let $\sigma$ be a regular hypersubstitution of type $\tau$ and let $\sigma(\mathscr{A})=$ $\left(A ;\left(\sigma\left(f_{i}\right)^{\mathscr{A}}\right)_{i \in I}\right)$. For a term $t \in W_{\tau}^{C}(X)$, denote by $t^{\sigma(\mathscr{A})}$ the term operation induced by $t$ in the algebra $\sigma(\mathscr{A})$, and by $\hat{\sigma}[t]^{\mathscr{A}}$ the term operation induced by $\hat{\sigma}[t]$ in the algebra $\mathscr{A}$. Then for every term $t \in W_{\tau}^{C}(X)$,

$$
\hat{\sigma}[t]^{A}=t^{\sigma(\mathscr{A})} .
$$

Lemma 2.6. Let $\sigma_{1}, \sigma_{2} \in \operatorname{Hyp}_{R}^{C}(\tau)$ and $\mathscr{A} \in P \operatorname{Alg}(\tau)$. Then $\sigma_{1}\left(\sigma_{2}(\mathscr{A})\right)=\left(\sigma_{2} \circ_{h} \sigma_{1}\right)(\mathscr{A})$.

Proof. We have

$$
\begin{aligned}
\sigma_{1}\left(\sigma_{2}(\mathscr{A})\right) & =\left(A ;\left(f_{i}^{\sigma_{1}\left(\sigma_{2}(\mathscr{A})\right)}\right)_{i \in I}\right) \\
& =\left(A ;\left(\sigma_{1}\left(f_{i}\right)^{\sigma_{2}(\mathscr{A})}\right)_{i \in I}\right) \quad \text { by Lemma } 2.5 \\
& =\left(A ;\left(\hat{\sigma}_{2}\left[\sigma_{1}\left(f_{i}\right)\right]^{\mathscr{A}}\right)_{i \in I}\right) \quad \text { by Lemma } 2.5 \\
& =\left(A ;\left(\left(\sigma_{2} \circ{ }_{h} \sigma_{1}\right)\left(f_{i}\right)^{\mathscr{A}}\right)_{i \in I}\right) \\
& =\left(\sigma_{2} \circ{ }_{h} \sigma_{1}\right)(\mathscr{A}) .
\end{aligned}
$$

(Remark that for the fundamental operations of the derived algebra $\sigma(\mathscr{A})$ we have $f_{i}^{\sigma(\mathscr{A})}=\sigma\left(f_{i}\right)^{\mathscr{A}}$. For $\sigma_{1}\left(\sigma_{2}(\mathscr{A})\right)$ this gives $f_{i}^{\sigma_{1}\left(\sigma_{2}(\mathscr{A})\right)}=\sigma_{1}\left(f_{i}\right)^{\sigma_{2}(\mathscr{A})}=\hat{\sigma}_{2}\left[\sigma_{1}\left(f_{i}\right)\right]^{\mathscr{A}}$ by Lemma 2.5.) Definition 2.7 (see [10]). A pair $t_{1} \approx t_{2} \in W_{\tau}^{C}(X)^{2}$ is called a strong identity in a partial algebra $\mathscr{A}$ (in symbols $\mathscr{A}={ }_{s} t_{1} \approx t_{2}$ ) if and only if $t_{1}^{\mathscr{A}}$ is defined whenever $t_{2}^{\mathscr{A}}$ is defined and conversely and $t_{1}^{A}=t_{2}^{A}$ on the common domain, that is, the induced partial term operations $t_{1}^{s}$ and $t_{2}^{s}$ are equal.

Let $K \subseteq P \operatorname{Alg}(\tau)$ be a class of partial algebras of type $\tau$ and $\Sigma \subseteq W_{\tau}^{C}(X)^{2}$. Consider the connection between $P \operatorname{Alg}(\tau)$ and $W_{\tau}^{C}(X)^{2}$ given by the following two operators:

$$
\operatorname{Id}^{s}: \mathscr{P}(P \operatorname{Alg}(\tau)) \longrightarrow \mathscr{P}\left(W_{\tau}^{C}(X)^{2}\right), \quad \operatorname{Mod}^{s}: \mathscr{P}\left(W_{\tau}^{C}(X)^{2}\right) \longrightarrow \mathscr{P}(P \operatorname{Alg}(\tau))
$$


with

$$
\begin{gathered}
\operatorname{Id}^{s} K:=\left\{s \approx t \in W_{\tau}^{C}(X)^{2} \mid \forall \mathscr{A} \in K\left(\underset{s}{\left.\right|_{s} s \approx t}\right)\right\}, \\
\operatorname{Mod}^{s} \Sigma:=\left\{\mathscr{A} \in P \operatorname{Alg}(\tau) \mid \forall s \approx t \in \Sigma\left(\mathscr{A}_{s}=s \approx t\right)\right\} .
\end{gathered}
$$

Clearly, the pair $\left(\operatorname{Mod}^{s}, \mathrm{Id}^{s}\right)$ is a Galois connection between $P \operatorname{Alg}(\tau)$ and $W_{\tau}^{C}(X)^{2}$. We have two closure operators $\operatorname{Mod}^{s} \mathrm{Id}^{s}$ and $\operatorname{Id}^{s} \operatorname{Mod}^{s}$ and their sets of fixed points.

Definition 2.8. Let $V \subseteq P \operatorname{Alg}(\tau)$ be a class of partial algebras of type $\tau$. The class $V$ is called a strong variety of partial algebras if $V=\operatorname{Mod}^{s} \mathrm{Id}^{s} V$.

Definition 2.9. Let $\mathscr{A}$ be a partial algebra of type $\tau$ and let $M$ be a submonoid of the monoid $\operatorname{Hyp}_{R}^{C}(\tau)$. Then, define

$$
\chi_{M}^{A}: \mathscr{P}(P \operatorname{Alg}(\tau)) \longrightarrow \mathscr{P}(P \operatorname{Alg}(\tau)), \quad \chi_{M}^{E}: \mathscr{P}\left(W_{\tau}^{C}(X)^{2}\right) \longrightarrow \mathscr{P}\left(W_{\tau}^{C}(X)^{2}\right)
$$

by

$$
\begin{gathered}
\chi_{M}^{A}(\mathscr{A}):=\{\sigma(\mathscr{A}) \mid \sigma \in M\}, \\
\chi_{M}^{E}[s \approx t]:=\{\hat{\sigma}[s] \approx \hat{\sigma}[t] \mid \sigma \in M\} .
\end{gathered}
$$

For $K \subseteq P \operatorname{Alg}(\tau)$ a class of partial algebras of type $\tau$ and for $\Sigma \subseteq W_{\tau}^{C}(X)^{2}$ we define $\chi_{M}^{A}(K):=\bigcup_{\mathscr{A} \in K} \chi_{M}^{A}(\mathscr{A})$ and $\chi_{M}^{E}[\Sigma]:=\bigcup_{s \approx t \in \Sigma} \chi_{M}^{E}[s \approx t]$.

Proposition 2.10. Let $\mathscr{A} \in P \operatorname{Alg}(\tau)$ and $s \approx t \in W_{\tau}^{C}(X)^{2}$. Then

$$
\left.\chi_{M}^{A}(\mathscr{A})\right|_{s} s \approx t \quad \text { iff }\left.\mathscr{A}\right|_{s} \chi_{M}^{E}[s \approx t] .
$$

Proof. We have

$$
\begin{aligned}
\left.\chi_{M}^{A}(\mathscr{A})\right|_{s} s \approx t & \Longleftrightarrow \forall \sigma \in M\left(\left.\sigma(\mathscr{A})\right|_{s} s \approx t\right) \\
& \Longleftrightarrow \forall \sigma \in M\left(s^{\sigma(\mathscr{A})}=t^{\sigma(\mathscr{A})}\right) \\
& \Longleftrightarrow \forall \sigma \in M\left(\hat{\sigma}[s]^{\mathscr{A}}=\hat{\sigma}[t]^{\mathscr{A}}\right) \\
& \Longleftrightarrow \forall \sigma \in M\left(\left.\mathscr{A}\right|_{s} \hat{\sigma}[s] \approx \hat{\sigma}[t]\right) \\
& \Longleftrightarrow \underset{s}{\mid} \chi_{M}^{E}[s \approx t] .
\end{aligned}
$$

Definition 2.11. Let $V$ be a strong variety of partial algebras of type $\tau$. Then $V$ is said to be $M$-solid if $\chi_{M}^{A}(V)=V$.

If $M=\operatorname{Hyp}_{R}^{C}(\tau)$, then $V$ is called solid. 


\section{3. $V$-proper hypersubstitutions}

Now we consider regular hypersubstitutions which preserve all strong identities of a strong variety of partial algebras.

Definition 3.1. Let $V$ be a strong variety of partial algebras of type $\tau$. A regular hypersubstitution $\sigma \in \operatorname{Hyp}_{R}^{C}(\tau)$ is called a $V$-proper hypersubstitution if for every $s \approx t \in \operatorname{Id}^{s} V$ one gets $\hat{\sigma}[s] \approx \hat{\sigma}[t] \in \operatorname{Id}^{s} V$.

We use $P(V)$ for the set of all $V$-proper hypersubstitutions of type $\tau$.

Proposition 3.2. The algebra $\left(P(V) ;{ }^{\circ}, \sigma_{\mathrm{id}}\right)$ is a submonoid of $\left(\operatorname{Hyp}_{R}^{C}(\tau) ;{ }^{\circ}, \sigma_{\mathrm{id}}\right)$.

Proof. Clearly, $\sigma_{\text {id }} \in P(V)$. If $\sigma_{1}, \sigma_{2} \in P(V)$, then for every $s \approx t \in \operatorname{Id}^{s} V$ we have $\hat{\sigma}_{2}[s] \approx$ $\hat{\sigma}_{2}[t] \in \operatorname{Id}^{s} V$ and $\hat{\sigma}_{1}\left[\hat{\sigma}_{2}[s]\right] \approx \hat{\sigma}_{1}\left[\hat{\sigma}_{2}[t]\right] \in \operatorname{Id}^{s} V$. This means that $\left(\hat{\sigma}_{1} \circ \hat{\sigma}_{2}\right)[s] \approx$ $\left(\hat{\sigma}_{1} \circ \hat{\sigma}_{2}\right)[t] \in \operatorname{Id}^{s} V$ and we get that $\left(\sigma_{1} \circ_{h} \sigma_{2}\right)^{\wedge}[s] \approx\left(\sigma_{1} \circ_{h} \sigma_{2}\right)^{\wedge}[t] \in \operatorname{Id}^{s} V$. Therefore $\sigma_{1} \circ_{h}$ $\sigma_{2} \in P(V)$, and we have that $P(V)$ is a submonoid of $\mathcal{H} y p_{R}^{c}(\tau)$.

Definition 3.3. Let $V$ be a strong variety of partial algebras of type $\tau$. Two regular hypersubstitutions $\sigma_{1}, \sigma_{2} \in \operatorname{Hyp}_{R}^{C}(\tau)$ are called $V$-equivalent if and only if $\sigma_{1}\left(f_{i}\right) \approx \sigma_{2}\left(f_{i}\right) \in \operatorname{Id}^{s} V$ for all $i \in I$. In this case we write $\sigma_{1} \sim_{V} \sigma_{2}$.

THeORem 3.4. Let $V$ be a strong variety of partial algebras of type $\tau$, and let $\sigma_{1}, \sigma_{2} \in$ $\operatorname{Hyp}_{R}^{C}(\tau)$. Then the following are equivalent:

(i) $\sigma_{1} \sim{ }_{V} \sigma_{2}$;

(ii) for all $t \in W_{\tau}^{C}(X)$ the equation $\hat{\sigma}_{1}[t] \approx \hat{\sigma}_{2}[t]$ is an identity from $\operatorname{Id}^{s} V$;

(iii) for all $\mathscr{A} \in V, \sigma_{1}(\mathscr{A})=\sigma_{2}(\mathscr{A})$.

Proof. (i) $\Rightarrow$ (ii). We will give a proof by induction on the complexity of the term $t$.

(1) If $t=x_{i} \in X$, then $\hat{\sigma}_{1}\left[x_{i}\right]=x_{i} \approx x_{i}=\hat{\sigma}_{2}\left[x_{i}\right] \in \operatorname{Id}^{s} V$.

(2) If $t=\varepsilon_{j}^{k}\left(t_{1}, \ldots, t_{k}\right)$ and if we assume that $\hat{\sigma}_{1}\left[t_{i}\right] \approx \hat{\sigma}_{2}\left[t_{i}\right] \in \operatorname{Id}^{s} V$ for all $1 \leq i \leq k$ (i.e., $\left.\hat{\sigma}_{1}\left[t_{i}\right]^{\mathscr{A}}\right|_{D}=\left.\hat{\sigma}_{2}\left[t_{i}\right]^{\mathscr{A}}\right|_{D}$ for all $\mathscr{A} \in V$ where $D$ is the intersection of all domains of $\hat{\sigma}_{1}\left[t_{i}\right]^{\mathcal{A}}$ and $\left.\hat{\sigma}_{2}\left[t_{i}\right]^{A} ; 1 \leq i \leq k\right)$, then

$$
\begin{aligned}
\left.\hat{\sigma}_{1}\left[\varepsilon_{j}^{k}\left(t_{1}, \ldots, t_{k}\right)\right]^{\mathscr{A}}\right|_{D} & =\left.\bar{S}_{n}^{k}\left(\varepsilon_{j}^{k}\left(x_{1}, \ldots, x_{k}\right), \hat{\sigma}_{1}\left[t_{1}\right], \ldots, \hat{\sigma}_{1}\left[t_{k}\right]\right)^{\mathscr{A}}\right|_{D} \\
& =\left.\varepsilon_{j}^{k}\left(\bar{S}_{n}^{k}\left(x_{1}, \hat{\sigma}_{1}\left[t_{1}\right], \ldots, \hat{\sigma}_{1}\left[t_{k}\right]\right), \ldots, \bar{S}_{n}^{k}\left(x_{k}, \hat{\sigma}_{1}\left[t_{1}\right], \ldots, \hat{\sigma}_{1}\left[t_{k}\right]\right)\right)^{\mathscr{A}}\right|_{D} \\
& =\left.\varepsilon_{j}^{k}\left(\hat{\sigma}_{1}\left[t_{1}\right], \ldots, \hat{\sigma}_{1}\left[t_{k}\right]\right)^{\mathscr{A}}\right|_{D}=\left.\hat{\sigma}_{1}\left[t_{j}\right]^{\mathscr{A}}\right|_{D} \\
& =\left.\hat{\sigma}_{2}\left[t_{j}\right]^{\mathscr{A}}\right|_{D}=\left.\varepsilon_{j}^{k}\left(\hat{\sigma}_{2}\left[t_{1}\right], \ldots, \hat{\sigma}_{2}\left[t_{k}\right]\right)^{\mathscr{A}}\right|_{D} \\
& =\left.\varepsilon_{j}^{k}\left(\bar{S}_{n}^{k}\left(x_{1}, \hat{\sigma}_{2}\left[t_{1}\right], \ldots, \hat{\sigma}_{2}\left[t_{k}\right]\right), \ldots, \bar{S}_{n}^{k}\left(x_{k}, \hat{\sigma}_{2}\left[t_{1}\right], \ldots, \hat{\sigma}_{2}\left[t_{k}\right]\right)\right)^{\mathscr{A}}\right|_{D} \\
& =\left.\bar{S}_{n}^{k}\left(\varepsilon_{j}^{k}\left(x_{1}, \ldots, x_{k}\right), \hat{\sigma}_{2}\left[t_{1}\right], \ldots, \hat{\sigma}_{2}\left[t_{k}\right]\right)^{\mathscr{A}}\right|_{D}=\left.\hat{\sigma}_{2}\left[\varepsilon_{j}^{k}\left(t_{1}, \ldots, t_{k}\right)\right]^{\mathscr{A}}\right|_{D} .
\end{aligned}
$$

(3) If $t=f_{i}\left(t_{1}, \ldots, t_{n_{i}}\right)$ and if we assume that $\hat{\sigma}_{1}\left[t_{j}\right] \approx \hat{\sigma}_{2}\left[t_{j}\right] \in \operatorname{Id}^{s} V$ for all $1 \leq j \leq n_{i}$ (i.e., $\left.\hat{\sigma}_{1}\left[t_{j}\right]^{\mathscr{A}}\right|_{D}=\left.\hat{\sigma}_{2}\left[t_{j}\right]^{\mathscr{A}}\right|_{D}$ for all $\mathscr{A} \in V$ where $D$ is the intersection of all domains of 
$\hat{\sigma}_{1}\left[t_{j}\right]^{\mathscr{A}}$ and $\left.\hat{\sigma}_{2}\left[t_{j}\right]^{\mathcal{A}} ; 1 \leq j \leq n_{i}\right)$, then

$$
\begin{aligned}
\left.\hat{\sigma}_{1}\left[f_{i}\left(t_{1}, \ldots, t_{n_{i}}\right)\right]^{\mathscr{A}}\right|_{D} & =\left.S_{n}^{n_{i}, \mathscr{A}}\left(\sigma_{1}\left(f_{i}\right)^{\mathscr{A}}, \hat{\sigma}_{1}\left[t_{1}\right]^{\mathscr{A}}, \ldots, \hat{\sigma}_{1}\left[t_{n_{i}}\right]^{\mathscr{A}}\right)\right|_{D} \\
& =S_{n}^{n_{i}, \mathscr{A}}\left(\sigma_{1}\left(f_{i}\right)^{\mathscr{A}},\left.\hat{\sigma}_{1}\left[t_{1}\right]^{\mathscr{A}}\right|_{D}, \ldots,\left.\hat{\sigma}_{1}\left[t_{n_{i}}\right]^{\mathscr{A}}\right|_{D}\right) \\
& =S_{n}^{n_{i}, \mathscr{A}}\left(\sigma_{1}\left(f_{i}\right)^{\mathscr{A}},\left.\hat{\sigma}_{2}\left[t_{1}\right]^{\mathscr{A}}\right|_{D}, \ldots,\left.\hat{\sigma}_{2}\left[t_{n_{i}}\right]^{\mathscr{A}}\right|_{D}\right) \\
& =S_{n}^{n_{i}, \mathscr{A}}\left(\sigma_{2}\left(f_{i}\right)^{\mathscr{A}},\left.\hat{\sigma}_{2}\left[t_{1}\right]^{\mathscr{A}}\right|_{D}, \ldots,\left.\hat{\sigma}_{2}\left[t_{n_{i}}\right]^{\mathscr{A}}\right|_{D}\right) \\
& =\left.S_{n}^{n_{i}, \mathscr{A}}\left(\sigma_{2}\left(f_{i}\right)^{\mathscr{A}}, \hat{\sigma}_{2}\left[t_{1}\right]^{\mathscr{A}}, \ldots, \hat{\sigma}_{2}\left[t_{n_{i}}\right]^{\mathscr{A}}\right)\right|_{D}=\left.\hat{\sigma}_{2}\left[f_{i}\left(t_{1}, \ldots, t_{n_{i}}\right)\right]^{\mathscr{A}}\right|_{D} .
\end{aligned}
$$

If $\left.\hat{\sigma}_{1}\left[t_{j}\right]^{\mathscr{A}}\right|_{D}$ is not in the domain of $\sigma_{1}\left(f_{i}\right)^{\mathscr{A}}$, then both sides are not defined.

(ii) $\Rightarrow$ (iii). We consider the term $t=f_{i}\left(x_{1}, \ldots, x_{n_{i}}\right)$ for $i \in I$. Then $\hat{\sigma}_{1}\left[f_{i}\left(x_{1}, \ldots, x_{n_{i}}\right)\right] \approx$ $\hat{\sigma}_{2}\left[f_{i}\left(x_{1}, \ldots, x_{n_{i}}\right)\right] \in \operatorname{Id}^{s} \mathscr{A}$ for all $\mathscr{A} \in V$ by (ii) and we get $\hat{\sigma}_{1}\left[f_{i}\left(x_{1}, \ldots, x_{n_{i}}\right)\right]^{\mathscr{A}}=\hat{\sigma}_{2}\left[f_{i}\left(x_{1}, \ldots\right.\right.$, $\left.\left.x_{n_{i}}\right)\right]^{\mathscr{A}}$ for all $i \in I$ and all $\mathscr{A} \in V$. Thus $\sigma_{1}(\mathscr{A})=\sigma_{2}(\mathscr{A})$.

(iii) $\Rightarrow$ (i). Here we have $\hat{\sigma}_{1}\left[f_{i}\left(x_{1}, \ldots, x_{n_{i}}\right)\right]^{\mathscr{A}}=\hat{\sigma}_{2}\left[f_{i}\left(x_{1}, \ldots, x_{n_{i}}\right)\right]^{\mathscr{A}}$ for all $i \in I$ and all $\mathscr{A} \in$ $V$. Therefore $\sigma_{1}\left(f_{i}\right) \approx \sigma_{2}\left(f_{i}\right) \in \mathrm{Id}^{s} \mathscr{A}$ for all $\mathscr{A} \in V$. So, $\sigma_{1} \sim_{V} \sigma_{2}$.

Proposition 3.5. Let $V$ be a strong variety of partial algebras of type $\tau$. Then the relation $\sim_{V}$ is a right congruence on $\operatorname{Hyp}_{R}^{C}(\tau)$.

Proof. Let $\sigma_{1} \sim_{V} \sigma_{2}$ and $\sigma \in \operatorname{Hyp}_{R}^{C}(\tau)$. By Theorem 3.4(ii) we have

$$
\left(\sigma_{1} \circ_{h} \sigma\right)\left(f_{i}\right)=\hat{\sigma}_{1}\left[\sigma\left(f_{i}\right)\right] \approx \hat{\sigma}_{2}\left[\sigma\left(f_{i}\right)\right]=\left(\sigma_{2} \circ_{h} \sigma\right)\left(f_{i}\right) \in \operatorname{Id}^{s} V
$$

So, $\sigma_{1}{ }^{\circ}{ }_{h} \sigma \sim_{V} \sigma_{2}{ }^{\circ}{ }_{h} \sigma$. This shows that $\sim_{V}$ is a right congruence.

In general, $\sim_{V}$ is not a left congruence. But if $V$ is solid, then it is a congruence.

Proposition 3.6. Let $V$ be a strong variety of partial algebras of type $\tau$. If $\sigma_{1} \sim_{V} \sigma_{2}$ and $\hat{\sigma}_{1}[s] \approx \hat{\sigma}_{1}[t] \in \operatorname{Id}^{s} V$, then $\hat{\sigma}_{2}[s] \approx \hat{\sigma}_{2}[t] \in \operatorname{Id}^{s} V$ when $\sigma_{1}, \sigma_{2} \in \operatorname{Hyp}_{R}^{C}(\tau)$ and $s, t \in W_{\tau}^{C}(X)$.

Proof. Assume that $\sigma_{1} \sim_{V} \sigma_{2}$ and $\hat{\sigma}_{1}[s] \approx \hat{\sigma}_{1}[t] \in \operatorname{Id}^{s} V$. By Theorem 3.4, we have $\hat{\sigma}_{1}[s] \approx$ $\hat{\sigma}_{2}[s] \in \operatorname{Id}^{s} V$ and $\hat{\sigma}_{1}[t] \approx \hat{\sigma}_{2}[t] \in \operatorname{Id}^{s} V$. Thus $\hat{\sigma}_{2}[s] \approx \hat{\sigma}_{2}[t] \in \operatorname{Id}^{s} V$.

As a corollary we get the following.

Corollary 3.7. The set $P(V)$ is a union of equivalence classes with respect to $\sim_{V}$.

Now we consider the equivalence class of the identity hypersubstitution.

Definition 3.8. A regular hypersubstitution $\sigma \in \operatorname{Hyp}_{R}^{C}(\tau)$ is called an inner hypersubstitution of a strong variety $V$ of partial algebras of type $\tau$ if for every $i \in I$,

$$
\hat{\sigma}\left[f_{i}\left(x_{1}, \ldots, x_{n_{i}}\right)\right] \approx f_{i}\left(x_{1}, \ldots, x_{n_{i}}\right) \in \operatorname{Id}^{s} V
$$

Let $P_{0}(V)$ be the set of all inner hypersubstitutions of $V$.

By definition, $P_{0}(V)$ is the equivalence class $\left[\sigma_{\mathrm{id}}\right]_{\sim_{V}}$. 
8 Unsolid and fluid strong varieties of partial algebras

Proposition 3.9. If $\sigma \in P_{0}(V)$, then $\hat{\sigma}[t] \approx t \in \operatorname{Id}^{s} V$ for $t \in W_{\tau}^{C}(X)$.

Proof. We will give a proof by induction on the complexity of the term $t$.

(i) If $t=x_{i} \in X$, then $\hat{\sigma}[t]=x_{i} \approx x_{i}=t \in \operatorname{Id}^{s} V$.

(ii) If $t=\varepsilon_{j}^{k}\left(t_{1}, \ldots, t_{k}\right)$ and if we assume that $\hat{\sigma}\left[t_{i}\right] \approx t_{i} \in \operatorname{Id}^{s} V$ for all $1 \leq i \leq k$ (i.e., $\left.\hat{\sigma}\left[t_{i}\right]^{\mathscr{A}}\right|_{D}=\left.t_{i}^{\mathscr{A}}\right|_{D}$ for all $\mathscr{A} \in V$ where $D$ is the intersection of all domains of $\hat{\sigma}\left[t_{i}\right]^{\mathscr{A}}$ and $t_{i}^{\mathscr{A}}$; $1 \leq i \leq k)$, then

$$
\begin{aligned}
\left.\hat{\sigma}\left[\varepsilon_{j}^{k}\left(t_{1}, \ldots, t_{k}\right)\right]^{\mathscr{A}}\right|_{D} & =\left.\bar{S}_{n}^{k}\left(\varepsilon_{j}^{k}\left(x_{1}, \ldots, x_{k}\right), \hat{\sigma}\left[t_{1}\right], \ldots, \hat{\sigma}\left[t_{k}\right]\right)^{\mathscr{A}}\right|_{D} \\
& =\left.\varepsilon_{j}^{k}\left(\bar{S}_{n}^{k}\left(x_{1}, \hat{\sigma}\left[t_{1}\right], \ldots, \hat{\sigma}\left[t_{k}\right]\right), \ldots, \bar{S}_{n}^{k}\left(x_{k}, \hat{\sigma}\left[t_{1}\right], \ldots, \hat{\sigma}\left[t_{k}\right]\right)\right)^{\mathscr{A}}\right|_{D} \\
& =\left.\varepsilon_{j}^{k}\left(\hat{\sigma}\left[t_{1}\right], \ldots, \hat{\sigma}\left[t_{k}\right]\right)^{\mathscr{A}}\right|_{D}=\left.\hat{\sigma}\left[t_{j}\right]^{\mathscr{A}}\right|_{D}=\left.t_{j}^{\mathscr{A}}\right|_{D} \\
& =\left.\varepsilon_{j}^{k}\left(t_{1}, \ldots, t_{k}\right)^{\mathscr{A}}\right|_{D}=\left.t^{\mathscr{A}}\right|_{D} .
\end{aligned}
$$

(iii) If $t=f_{i}\left(t_{1}, \ldots, t_{n_{i}}\right)$ and if we assume that $\hat{\sigma}\left[t_{j}\right] \approx t_{j} \in \operatorname{Id}^{s} V$ for all $1 \leq j \leq n_{i}$ (i.e., $\left.\hat{\sigma}\left[t_{j}\right]^{\mathscr{A}}\right|_{D}=\left.t_{j}^{\mathscr{A}}\right|_{D}$ for all $\mathscr{A} \in V$ where $D$ is the intersection of all domains of $\hat{\sigma}\left[t_{j}\right]^{\mathscr{A}}$ and $\left.t_{j}^{A} ; 1 \leq j \leq n_{i}\right)$, then

$$
\begin{aligned}
\left.\hat{\sigma}\left[f_{i}\left(t_{1}, \ldots, t_{n_{i}}\right)\right]^{\mathscr{A}}\right|_{D} & =\left.S_{n}^{n_{i}, \mathscr{A}}\left(\sigma\left(f_{i}\right)^{\mathscr{A}}, \hat{\sigma}\left[t_{1}\right]^{\mathscr{A}}, \ldots, \hat{\sigma}\left[t_{n_{i}}\right]^{\mathscr{A}}\right)\right|_{D} \\
& =S_{n}^{n_{i}, \mathscr{A}}\left(\sigma\left(f_{i}\right)^{\mathscr{A}},\left.\hat{\sigma}\left[t_{1}\right]^{\mathscr{A}}\right|_{D}, \ldots,\left.\hat{\sigma}\left[t_{n_{i}}\right]^{\mathscr{A}}\right|_{D}\right) \\
& =S_{n}^{n_{i}, \mathscr{A}}\left(\sigma\left(f_{i}\right)^{\mathscr{A}},\left.t_{1}^{\mathscr{A}}\right|_{D}, \ldots,\left.t_{n_{i}}\right|_{D}\right) \\
& =S_{n}^{n_{i}, \mathscr{A}}\left(f_{i}^{A},\left.t_{1}^{\mathscr{A}}\right|_{D}, \ldots,\left.t_{n_{i}}^{A}\right|_{D}\right) \quad \text { by } \sigma \in P_{0}(V) \\
& =\left.S_{n}^{n_{i}, \mathscr{A}}\left(f_{i}^{A}, t_{1}^{A}, \ldots, t_{n_{i}}\right)\right|_{D}=\left.f_{i}\left(t_{1}, \ldots, t_{n_{i}}\right)^{\mathscr{A}}\right|_{D} .
\end{aligned}
$$

If $\left.\hat{\sigma}\left[t_{j}\right]^{\mathscr{A}}\right|_{D}$ is not in the domain of $\sigma\left(f_{i}\right)^{\mathscr{A}}$, then both sides are not defined.

Proposition 3.10. The algebra $\left(P_{0}(V) ;{ }^{\circ} h, \sigma_{\mathrm{id}}\right)$ is a submonoid of $\left(P(V) ; \circ_{h}, \sigma_{\mathrm{id}}\right)$.

Proof. Clearly, $\sigma_{\text {id }} \in P_{0}(V)$. Assume that $\sigma_{1}, \sigma_{2} \in P_{0}(V)$. Then

$$
\begin{aligned}
\left(\sigma_{1} \circ{ }_{h} \sigma_{2}\right)^{\Upsilon}\left[f_{i}\left(x_{1}, \ldots, x_{n_{i}}\right)\right] & =\hat{\sigma}_{1}\left[\hat{\sigma}_{2}\left[f_{i}\left(x_{1}, \ldots, x_{n_{i}}\right)\right]\right] \\
& \approx \hat{\sigma}_{1}\left[f_{i}\left(x_{1}, \ldots, x_{n_{i}}\right)\right] \quad \text { by Proposition } 3.9 \\
& \approx f_{i}\left(x_{1}, \ldots, x_{n_{i}}\right) \quad \text { by Proposition } 3.9 \\
& \in \operatorname{Id}^{s} V .
\end{aligned}
$$


Therefore $\sigma_{1}{ }^{\circ}{ }_{h} \sigma_{2} \in P_{0}(V)$. Thus $P_{0}(V)$ is a monoid. By Proposition 3.9, we have $P_{0}(V) \subseteq P(V)$. So, the algebra $\left(P_{0}(V) ;{ }^{\circ}, \sigma_{\text {id }}\right)$ is a submonoid of $\left(P(V) ;{ }^{\circ} h, \sigma_{\text {id }}\right)$.

Now we show that the compatibility condition from the definition of a closed homomorphism for partial algebras (see [2]) transfers from fundamental operations to arbitrary term operations.

Lemma 3.11. Let $\mathscr{A} \in P \operatorname{Alg}(\tau)$ and let $t^{\mathscr{A}}$ be the $n$-ary term operation on $A$ induced by the $n$-ary term $t \in W_{\tau}^{C}(X)$. If $\mathscr{B} \in P \operatorname{Alg}(\tau)$ and if $\varphi: \mathscr{A} \rightarrow \mathscr{B}$ is a surjective closed homomorphism, then for all $a_{1}, \ldots, a_{n} \in A$,

$$
\varphi\left(t^{\mathscr{A}}\left(a_{1}, \ldots, a_{n}\right)\right)=t^{\mathscr{P}}\left(\varphi\left(a_{1}\right), \ldots, \varphi\left(a_{n}\right)\right) .
$$

Proof. We will give a proof by induction on the complexity of the term $t$.

(i) If $t=x_{i} \in X_{n}$, then $t^{\mathscr{A}}=x_{i}^{\mathscr{A}}=e_{i}^{n, A}$ and $t^{\mathscr{P}}=x_{i}^{\mathscr{B}}=e_{i}^{n, B}$. We get $\operatorname{dom} t^{\mathscr{A}}=A^{n}$ and $\operatorname{dom} t^{\mathscr{R}}=B^{n}$. Since $\varphi$ is a surjective, we have

$$
\begin{aligned}
\left(a_{1}, \ldots, a_{n}\right) \in \operatorname{dom} t^{\mathscr{A}} & \Longleftrightarrow\left(\varphi\left(a_{1}\right), \ldots, \varphi\left(a_{n}\right)\right) \in \operatorname{dom} t^{\mathscr{P}}, \\
\varphi\left(t^{\mathscr{A}}\left(a_{1}, \ldots, a_{n}\right)\right) & =\varphi\left(e_{i}^{n, A}\left(a_{1}, \ldots, a_{n}\right)\right)=\varphi\left(a_{i}\right) \\
& =e_{i}^{n, B}\left(\varphi\left(a_{1}\right), \ldots, \varphi\left(a_{n}\right)\right) \\
& =t^{\Re}\left(\varphi\left(a_{1}\right), \ldots, \varphi\left(a_{n}\right)\right) .
\end{aligned}
$$

(ii) If $t=\varepsilon_{j}^{k}\left(t_{1}, \ldots, t_{k}\right)$ and if we assume that

$$
\left(a_{1}, \ldots, a_{n}\right) \in \operatorname{dom} t_{i}^{A} \Longleftrightarrow\left(\varphi\left(a_{1}\right), \ldots, \varphi\left(a_{n}\right)\right) \in \operatorname{dom} t_{i}^{\Re}
$$

and $\varphi\left(t_{i}^{\mathscr{A}}\left(a_{1}, \ldots, a_{n}\right)\right)=t_{i}^{\Re}\left(\varphi\left(a_{1}\right), \ldots,\left(a_{n}\right)\right)$ for all $1 \leq i \leq k$, then

$$
\begin{aligned}
&\left(a_{1}, \ldots, a_{n}\right) \in \operatorname{dom} t^{\mathscr{A}} \Longleftrightarrow\left(a_{1}, \ldots, a_{n}\right) \in \operatorname{dom} \varepsilon_{j}^{k}\left(t_{1}, \ldots, t_{k}\right)^{\mathscr{A}} \\
& \Longleftrightarrow\left(a_{1}, \ldots, a_{n}\right) \in \operatorname{dom} t_{j}^{\mathscr{A}} \\
& \Longleftrightarrow\left(\varphi\left(a_{1}\right), \ldots, \varphi\left(a_{n}\right)\right) \in \operatorname{dom} t_{j}^{\mathscr{P}} \\
& \Longleftrightarrow\left(\varphi\left(a_{1}\right), \ldots, \varphi\left(a_{n}\right)\right) \in \operatorname{dom} \varepsilon_{j}^{k}\left(t_{1}, \ldots, t_{k}\right)^{\mathscr{B}} \\
& \Longleftrightarrow\left(\varphi\left(a_{1}\right), \ldots, \varphi\left(a_{n}\right)\right) \in \operatorname{dom} t^{\mathscr{B}}, \\
& \varphi\left(t^{\mathscr{A}}\left(a_{1}, \ldots, a_{n}\right)\right)=\varphi\left(\varepsilon_{j}^{k}\left(t_{1}, \ldots, t_{k}\right)^{\mathscr{A}}\left(a_{1}, \ldots, a_{n}\right)\right) \\
&=\varphi\left(t_{j}^{\mathscr{A}}\left(a_{1}, \ldots, a_{n}\right)\right)=t_{j}^{\mathscr{P}}\left(\varphi\left(a_{1}\right), \ldots, \varphi\left(a_{n}\right)\right) \\
&=\varepsilon_{j}^{k}\left(t_{1}, \ldots, t_{k}\right)^{\mathscr{B}}\left(\varphi\left(a_{1}\right), \ldots, \varphi\left(a_{n}\right)\right)=t^{\mathscr{B}}\left(\varphi\left(a_{1}\right), \ldots, \varphi\left(a_{n}\right)\right) .
\end{aligned}
$$


(iii) If $t=f_{i}\left(t_{1}, \ldots, t_{n_{i}}\right)$ and if we assume that $\left(a_{1}, \ldots, a_{n}\right) \in \operatorname{dom} t_{j}^{\mathscr{A}}$ if and only if $\left(\varphi\left(a_{1}\right), \ldots, \varphi\left(a_{n}\right)\right) \in \operatorname{dom} t_{j}^{\Im}$ and $\varphi\left(t_{j}^{\Im}\left(a_{1}, \ldots, a_{n}\right)\right)=t_{j}^{\Re}\left(\varphi\left(a_{1}\right), \ldots,\left(a_{n}\right)\right)$ for all $1 \leq j \leq n_{i}$, then

$$
\begin{gathered}
\left(a_{1}, \ldots, a_{n}\right) \in \operatorname{dom} t^{\mathscr{A}} \Longleftrightarrow\left(a_{1}, \ldots, a_{n}\right) \in \bigcap_{i=1}^{n_{i}} \operatorname{dom} t_{i}^{\unlhd}, \\
t_{i}^{\mathscr{A}}\left(a_{1}, \ldots, a_{n}\right)=b_{i} \quad\left(i=1, \ldots, n_{i}\right) \\
\left(b_{1}, \ldots, b_{n_{i}}\right) \in \operatorname{dom} f_{i}^{A}
\end{gathered}
$$

and we get $t^{\mathscr{A}}\left(a_{1}, \ldots, a_{n}\right)=f_{i}^{A}\left(b_{1}, \ldots, b_{n_{i}}\right)$.

By assumption, $\left(a_{1}, \ldots, a_{n}\right) \in \bigcap_{i=1}^{n_{i}} \operatorname{dom} t_{i}^{\not A}$ if and only if $\left(\varphi\left(a_{1}\right), \ldots, \varphi\left(a_{n}\right)\right) \in \bigcap_{i=1}^{n_{i}} \operatorname{dom} t_{i}^{\mathscr{P}}$ and $\varphi\left(t_{i}^{\circledR A}\left(a_{1}, \ldots, a_{n}\right)\right)=\varphi\left(b_{i}\right)=t_{i}^{\oiint_{P}}\left(\varphi\left(a_{1}\right), \ldots, \varphi\left(a_{n}\right)\right) ; i=1, \ldots, n_{i}$.

Since $\varphi$ is a closed homomorphism, we get

$$
\left(b_{1}, \ldots, b_{n_{i}}\right) \in \operatorname{dom} f_{i}^{A} \Longleftrightarrow\left(\varphi\left(b_{1}\right), \ldots, \varphi\left(b_{n_{i}}\right)\right) \in \operatorname{dom} f_{i}^{B}
$$

From $\left(\varphi\left(a_{1}\right), \ldots, \varphi\left(a_{n}\right)\right) \in \bigcap_{i=1}^{n_{i}} \operatorname{dom} t_{i}^{\mathscr{P}}$ and $t_{i}^{\mathscr{P}}\left(\varphi\left(a_{1}\right), \ldots, \varphi\left(a_{n}\right)\right)=\varphi\left(b_{i}\right)\left(i=1, \ldots, n_{i}\right)$ and $\left(\varphi\left(b_{1}\right), \ldots, \varphi\left(b_{n_{i}}\right)\right) \in \operatorname{dom} f_{i}^{B}$ we get $\left(\varphi\left(a_{1}\right), \ldots, \varphi\left(a_{n}\right)\right) \in \operatorname{dom} f_{i}\left(t_{1}, \ldots, t_{n_{i}}\right)^{\mathscr{B}}$.

Therefore,

$$
\begin{aligned}
\left(a_{1}, \ldots, a_{n}\right) \in \operatorname{dom} t^{\mathscr{A}} \Longleftrightarrow & \left(\varphi\left(a_{1}\right), \ldots, \varphi\left(a_{n}\right)\right) \in \operatorname{dom} t^{\mathscr{P}}, \\
\varphi\left(t^{\mathscr{A}}\left(a_{1}, \ldots, a_{n}\right)\right) & =\varphi\left(f_{i}^{A}\left(b_{1}, \ldots, b_{n_{i}}\right)\right) \\
& =f_{i}^{B}\left(\varphi\left(b_{1}\right), \ldots, \varphi\left(b_{n_{i}}\right)\right) \\
& =t^{\Re}\left(\varphi\left(a_{1}\right), \ldots, \varphi\left(a_{n}\right)\right) .
\end{aligned}
$$

Lemma 3.12. Let $\mathscr{A}, \mathscr{B} \in P \operatorname{Alg}(\tau)$ and $\sigma \in \operatorname{Hyp}_{R}^{C}(\tau)$. If $h: \mathscr{A} \rightarrow \mathscr{B}$ is a surjective closed homomorphism, then $h: \sigma(\mathscr{A}) \rightarrow \sigma(\mathscr{B})$ is a closed homomorphism.

Proof. From Lemma 3.11, for the term $\sigma\left(f_{i}\right)$ we have $h\left(f_{i}^{\sigma(\mathscr{A})}\left(a_{1}, \ldots, a_{n}\right)\right)=h\left(\sigma\left(f_{i}\right)^{\mathscr{A}}\left(a_{1}\right.\right.$, $\left.\left.\ldots, a_{n}\right)\right)=\sigma\left(f_{i}\right)^{\mathscr{B}}\left(h\left(a_{1}\right), \ldots, h\left(a_{n}\right)\right)=f_{i}^{\sigma(\mathscr{B})}\left(h\left(a_{1}\right), \ldots, h\left(a_{n}\right)\right)$. This shows that $h: \sigma(\mathscr{A}) \rightarrow$ $\sigma(\mathscr{B})$ is a closed homomorphism.

Lemma 3.13. Let $\mathscr{A}, \mathscr{B} \in P \operatorname{Alg}(\tau)$ and $\sigma \in \operatorname{Hyp}_{R}^{C}(\tau)$. If $f: \mathscr{A} \rightarrow \mathscr{B}$ is an isomorphism, then $f$ is also an isomorphism from $\sigma(\mathscr{A})$ to $\sigma(\mathscr{B})$.

Proof. Since $f: \mathscr{A} \rightarrow \mathscr{B}$ is bijective, the mapping $f: \sigma(\mathscr{A}) \rightarrow \sigma(\mathscr{B})$ is also bijective because partial algebras and their derived algebras have the same universes and by Lemma 3.12, we have $\sigma(\mathscr{A}) \cong \sigma(\mathscr{B})$. 
Definition 3.14. Let $V$ be a strong variety of partial algebras of type $\tau$ and $\sigma_{1}, \sigma_{2} \in \operatorname{Hyp}_{R}^{C}(\tau)$. Then, define

$$
\sigma_{1} \sim_{V-\text { iso }} \sigma_{2} \quad \text { iff } \sigma_{1}(\mathscr{A}) \cong \sigma_{2}(\mathscr{A}) \forall \mathscr{A} \in V
$$

Clearly, $\sim_{V} \subseteq \sim_{V \text {-iso }}$. If $V=P \operatorname{Alg}(\tau)$, then, use $\sim_{\text {iso }}$ instead of $\sim_{P \operatorname{Alg}(\tau) \text {-iso }}$.

Proposition 3.15. Let $V$ be a strong variety of partial algebras of type $\tau$. Then

(i) the relation $\sim_{V \text {-iso }}$ is a right congruence on $\operatorname{Hyp}_{R}^{C}(\tau)$;

(ii) if $V$ is a solid variety, then $\sim_{V-\text { iso }}$ is a congruence on $\operatorname{Hyp}_{R}^{C}(\tau)$.

Proof. (i) Let $\sigma_{1} \sim_{V \text {-iso }} \sigma_{2}$ and $\sigma \in \operatorname{Hyp}_{R}^{C}(\tau)$. Then $\sigma_{1}(\mathscr{A}) \cong \sigma_{2}(\mathscr{A})$ and $\sigma\left(\sigma_{1}(\mathscr{A})\right) \cong$ $\sigma\left(\sigma_{2}(\mathscr{A})\right)$ for all $\mathscr{A} \in V$ by Lemma 3.13. We have

$$
\left(\sigma_{1} \circ_{h} \sigma\right)(\mathscr{A})=\sigma\left(\sigma_{1}(\mathscr{A})\right) \cong \sigma\left(\sigma_{2}(\mathscr{A})\right)=\left(\sigma_{2} \circ_{h} \sigma\right)(\mathscr{A})
$$

So, $\sigma_{1}{ }^{\circ}{ }_{h} \sigma \sim_{V-\text { iso }} \sigma_{2}{ }^{\circ}{ }_{h} \sigma$. This shows that $\sim_{V-\text { iso }}$ is a right congruence.

(ii) Assume that $V$ is solid. Then $\sigma(\mathscr{A}) \in V$ for all $\sigma \in \operatorname{Hyp}_{R}^{C}(\tau)$ for $\mathscr{A} \in V . \sigma_{1} \sim_{V \text {-iso }}$ $\sigma_{2}$ implies that $\sigma_{1}(\sigma(\mathscr{A})) \cong \sigma_{2}(\sigma(\mathscr{A}))$ for all $\mathscr{A} \in V$. We have

$$
\left(\sigma \circ{ }_{h} \sigma_{1}\right)(\mathscr{A})=\sigma_{1}(\sigma(\mathscr{A})) \cong \sigma_{2}(\sigma(\mathscr{A}))=\left(\sigma \circ{ }_{h} \sigma_{2}\right)(\mathscr{A}) .
$$

So, $\sigma \circ_{h} \sigma_{1} \sim_{V \text {-iso }} \sigma \circ_{h} \sigma_{2}$. This shows that $\sim_{V-\text { iso }}$ is a left congruence and (i) shows that it is a congruence.

Proposition 3.16. If $V=P \operatorname{Alg}(\tau)$, then $\sim_{\text {iso }}$ is a congruence on $\operatorname{Hyp}_{R}^{C}(\tau)$.

Proof. Since $V=P \operatorname{Alg}(\tau)$ is a solid variety, the claim follows from Proposition 3.15(ii).

Proposition 3.17. The equivalence class $P_{0}^{V-\text { iso }}(V)=\left[\sigma_{\text {id }}\right]_{\sim_{V-\text { iso }}}$ is the submonoid of $\left(\operatorname{Hyp}_{R}^{C}(\tau) ;{ }_{h}, \sigma_{\text {id }}\right)$.

Proof. Clearly, $\sigma_{\text {id }} \in P_{0}^{V-\text { iso }}(V)$. Next, we will show that $P_{0}^{V-\text { iso }}(V)$ is closed under the operation $\circ_{h}$. Let $\sigma_{1}, \sigma_{2} \in P_{0}^{V-\text { iso }}(V)$. Then $\sigma_{1} \sim_{V-\text { iso }} \sigma_{\text {id }}$ and $\sigma_{2} \sim_{V-\text { iso }} \sigma_{\text {id }}$ implies that $\sigma_{1}(\mathscr{A}) \cong \mathscr{A}$ and $\sigma_{2}(\mathscr{A}) \cong \mathscr{A}$ for all $\mathscr{A} \in V$.

We have

$$
\begin{aligned}
\left(\sigma_{1} \circ_{h} \sigma_{2}\right)(\mathscr{A}) & =\sigma_{2}\left(\sigma_{1}(\mathscr{A})\right) \quad \text { by Lemma } 2.6 \\
& \cong \sigma_{2}(\mathscr{A}) \quad \text { by } \sigma_{1} \in P_{0}^{V-\text { iso }}(V) \\
& \cong \mathscr{A} \quad \text { by } \sigma_{2} \in P_{0}^{V-\text { iso }}(V) .
\end{aligned}
$$

Then $\left(\sigma_{1}{ }^{\circ} \sigma_{2}\right) \sim_{V \text {-iso }} \sigma_{\text {id }}$. Therefore $\sigma_{1}{ }^{\circ}{ }_{h} \sigma_{2} \in P_{0}^{V-\text { iso }}(V)$. So, $P_{0}^{V-\text { iso }}(V)$ is a submonoid of $\mathcal{H} y p_{R}^{C}(\tau)$.

Proposition 3.18. Let $V$ be a strong variety of partial algebras of type $\tau, s \approx t \in \mathrm{Id}^{s} V$ for $s, t \in W_{\tau}^{C}\left(X_{n}\right)$ and let $\sigma_{1}, \sigma_{2} \in \operatorname{Hyp}_{R}^{C}(\tau)$. If $\sigma_{1} \sim_{V-\text { iso }} \sigma_{2}$ and $\hat{\sigma}_{1}[s] \approx \hat{\sigma}_{1}[t] \in \operatorname{Id}^{s} V$, then $\hat{\sigma}_{2}[s] \approx \hat{\sigma}_{2}[t] \in \operatorname{Id}^{s} V$. 
Proof. Assume that $\sigma_{1} \sim_{V \text {-iso }} \sigma_{2}$ and $\hat{\sigma}_{1}[s] \approx \hat{\sigma}_{1}[t] \in \operatorname{Id}^{s} V$. Then $\sigma_{1}(\mathscr{A}) \cong \sigma_{2}(\mathscr{A})$ for all $\mathscr{A} \in V$. We get that there is an isomorphism $\varphi$ from $\sigma_{1}(\mathscr{A})$ to $\sigma_{2}(\mathscr{A})$. Let $b_{1}, \ldots, b_{n} \in A$. Then there are elements $a_{1}, \ldots, a_{n} \in A$ such that $\varphi\left(a_{1}\right)=b_{1}, \ldots, \varphi\left(a_{n}\right)=b_{n}$.

We have

$$
\begin{aligned}
& \operatorname{dom}\left(\hat{\sigma}_{2}[s]^{\mathscr{A}}\right)=\left\{\left(b_{1}, \ldots, b_{n}\right) \mid \hat{\sigma}_{2}[s]^{\mathscr{A}}\left(b_{1}, \ldots, b_{n}\right) \exists\right\} \\
&=\left\{\left(b_{1}, \ldots, b_{n}\right) \mid \hat{\sigma}_{2}[s]^{\mathscr{A}}\left(\varphi\left(a_{1}\right), \ldots, \varphi\left(a_{n}\right)\right) \exists\right\} \\
&=\left\{\left(b_{1}, \ldots, b_{n}\right) \mid \varphi\left(\hat{\sigma}_{1}[s]^{\mathscr{A}}\left(a_{1}, \ldots, a_{n}\right)\right) \exists\right\} \\
&=\left\{\left(b_{1}, \ldots, b_{n}\right) \mid \varphi\left(\hat{\sigma}_{1}[t]^{\mathscr{A}}\left(a_{1}, \ldots, a_{n}\right)\right) \exists\right\} \\
& \text { since } \varphi \text { is an isomorphism from } \sigma_{1}(\mathscr{A}) \text { to } \sigma_{2}(\mathscr{A}) \\
&=\left\{\left(b_{1}, \ldots, b_{n}\right) \mid \hat{\sigma}_{2}[t]^{\mathscr{A}}\left(\varphi\left(a_{1}\right), \ldots, \varphi\left(\hat{\sigma}_{1}[t] \in \operatorname{Id}^{s} \mathscr{A} \quad \forall \mathscr{A} \in V\right\}\right.\right. \\
&\left.\hat{\sigma}_{2}[s]^{\mathscr{A}}\left(b_{1}, \ldots, b_{n}\right) \mid \hat{\sigma}_{2}[t]^{\mathscr{A}}\left(b_{1}, \ldots, b_{n}\right) \exists\right\}=\operatorname{dom}\left(\hat{\sigma}_{2}[t]^{\mathscr{A}}\right), \\
&=\hat{\sigma}_{2}[s]^{\mathscr{A}}\left(\varphi\left(a_{1}\right), \ldots, \varphi\left(a_{n}\right)\right)=\varphi\left(\hat{\sigma}_{1}[s]^{\mathscr{A}}\left(a_{1}, \ldots, a_{n}\right)\right) \\
&=\varphi\left(\hat{\sigma}_{1}[t]^{\mathscr{A}}\left(a_{1}, \ldots, a_{n}\right)\right)=\hat{\sigma}_{2}[t]^{\mathscr{A}}\left(\varphi\left(a_{1}\right), \ldots, \varphi\left(a_{n}\right)\right) \\
&=\hat{\sigma}_{2}[t]^{\mathscr{A}}\left(b_{1}, \ldots, b_{n}\right) .
\end{aligned}
$$

Then $\hat{\sigma}_{2}[s] \approx \hat{\sigma}_{2}[t] \in \operatorname{Id}^{s} \mathscr{A}$ for all $\mathscr{A} \in V$. So, $\hat{\sigma}_{2}[s] \approx \hat{\sigma}_{2}[t] \in \operatorname{Id}^{s} V$.

As a corollary we get the following.

Corollary 3.19. The set $P(V)$ is a union of equivalence classes with respect to $\sim_{V-\text { iso. }}$.

Remark 3.20. $P_{0}(V) \subseteq P_{0}^{V-\text { iso }}(V) \subseteq P(V)$.

\section{Unsolid and fluid strong varieties}

For a solid strong variety every strong identity is closed under all hypersubstitutions. At the other extreme is the case where the strong identities are closed only under the identity hypersubstitution.

Definition 4.1. A strong variety $V$ of partial algebras of type $\tau$ is said to be unsolid if $P(V)=P_{0}(V)$ and $V$ is said to be completely unsolid if $P(V)=P_{0}(V)=\left\{\sigma_{\text {id }}\right\}$.

Definition 4.2. A strong variety $V$ of partial algebras of type $\tau$ is said to be iso-unsolid if $P(V)=P_{0}^{V \text {-iso }}(V)$ and $V$ is said to be completely iso-unsolid if $P(V)=P_{0}^{V \text {-iso }}(V)=\left\{\sigma_{\mathrm{id}}\right\}$.

Proposition 4.3. Let $V$ be a strong variety of partial algebras of type $\tau$. Then

(i) if $V$ is unsolid, then $V$ is iso-unsolid;

(ii) $V$ is completely unsolid if and only if $V$ is completely iso-unsolid. 
Proof. (i) The claim follows from the definitions and Remark 3.20.

(ii) If $V$ is completely unsolid, then $V$ is completely iso-unsolid by Remark 3.20. Conversely, assume that $V$ is completely iso-unsolid. Then $P(V)=P_{0}^{V-\text { iso }}(V)=\left\{\sigma_{\text {id }}\right\}$. Since $P_{0}(V) \subseteq P(V)$ and $P(V) \neq \varnothing$, we get $P_{0}(V)=\left\{\sigma_{\text {id }}\right\}$. So, $V$ is completely unsolid.

Definition 4.4. A strong variety $V$ of partial algebras of type $\tau$ is said to be fluid if, for every partial algebra $\mathscr{A} \in V$ and every regular hypersubstitution $\sigma \in \operatorname{Hyp}_{R}^{C}(\tau)$, there holds

$$
\sigma(\mathscr{A}) \in V \Longrightarrow \sigma(\mathscr{A}) \cong \mathscr{A} \text {. }
$$

We denote by $\sigma(V)$ the class of all algebras $\sigma(\mathscr{A})$ with $\mathscr{A} \in V$. As an easy consequence of the definition we have the following result.

Proposition 4.5. If a strong variety $V$ of partial algebras of type $\tau$ is fluid, then for every regular hypersubstitution $\sigma \in \operatorname{Hyp}_{R}^{C}(\tau)$, there holds

$$
\sigma(V) \subseteq V \Longrightarrow \forall \mathscr{A} \in V(\sigma(\mathscr{A}) \cong \mathscr{A})
$$

Proposition 4.6. Let $V$ be a strong variety of partial algebras of type $\tau$. Then for all $\sigma \in$ $\operatorname{Hyp}_{R}^{C}(\tau), \sigma(V) \subseteq V$ if and only if $\sigma \in P(V)$.

Proof. Assume that $\sigma(V) \subseteq V$. Let $s \approx t \in \operatorname{Id}^{s} V$. Then $\operatorname{Id}^{s} V \subseteq \operatorname{Id}^{s} \sigma(V)$ and we have $s \approx$ $t \in \operatorname{Id}^{s} \sigma(V)$. So, $\hat{\sigma}[s] \approx \hat{\sigma}[t] \in \operatorname{Id}^{s} V$ by Proposition 2.10. Therefore $\sigma \in P(V)$. Conversely, we assume that $\sigma \in P(V)$. Let $\mathscr{A} \in \sigma(V)$ and $s \approx t \in \operatorname{Id}^{s} V$. Then $\hat{\sigma}[s] \approx \hat{\sigma}[t] \in \operatorname{Id}^{s} V$ by $\sigma \in P(V)$ and $s \approx t \in \operatorname{Id}^{s} \sigma(V)$ by Proposition 2.10. Since $\mathscr{A} \in \sigma(V)$ we have $s \approx t \in \operatorname{Id}^{s} \mathscr{A}$ and $\mathscr{A} \in V$. So, $\sigma(V) \subseteq V$.

This shows that if a strong variety $V$ of partial algebras of type $\tau$ is fluid, then for every regular hypersubstitution $\sigma \in \operatorname{Hyp}_{R}^{C}(\tau)$, there holds

$$
\sigma \in P(V) \Longrightarrow \forall \mathscr{A} \in V(\sigma(\mathscr{A}) \cong \mathscr{A})
$$

Proposition 4.7. Let $V$ be a fluid strong variety of partial algebras of type $\tau$. Then $P(V)=$ $\left[\sigma_{\text {id }}\right]_{\sim_{V-\text { iso }}}$.

Proof. Let $\sigma \in P(V)$. Then $\hat{\sigma}[s] \approx \hat{\sigma}[t] \in \operatorname{Id}^{s} V$ for all $s \approx t \in \operatorname{Id}^{s} V$ implies that $\hat{\sigma}[s] \approx$ $\hat{\sigma}[t] \in \operatorname{Id}^{s} \mathscr{A}$ for all $\mathscr{A} \in V$. By Proposition 2.10, we have $s \approx t \in \operatorname{Id}^{s} \sigma(\mathscr{A})$. So, $\sigma(\mathscr{A}) \in V$ for all $\mathscr{A} \in V$ and for all $\sigma \in \operatorname{Hyp}_{R}^{C}(\tau)$. Since $V$ is fluid, we have $\sigma(\mathscr{A}) \cong \mathscr{A}$ and this implies that $\sigma \sim_{V \text {-iso }} \sigma_{\text {id }}$. Therefore $\sigma \in\left[\sigma_{\text {id }}\right]_{\sim_{V-\text { iso }}}$. Thus $P(V) \subseteq\left[\sigma_{\text {id }}\right]_{\sim_{V-\text { iso }}}$ but $\left[\sigma_{\text {id }}\right]_{\sim_{V-\text { iso }}} \subseteq P(V)$. So, $P(V)=\left[\sigma_{\text {id }}\right]_{\sim_{V-\text { iso }}}$.

Proposition 4.8. Let $V$ be solid variety of partial algebras of type $\tau$. Then $V$ is fluid if and only if $P(V)=\left[\sigma_{\text {id }}\right]_{\sim_{V-\text { iso }}}$.

Proof. By Proposition 4.7, we have that if $V$ is fluid, then $P(V)=\left[\sigma_{\text {id }}\right]_{\sim_{V \text {-iso }}}$. Conversely, we assume that $P(V)=\left[\sigma_{\text {id }}\right]_{\sim_{V-\text { iso }}}$. Let $\sigma \in \operatorname{Hyp}_{R}^{C}(\tau)$. Since $V$ is solid, we get $\sigma(\mathscr{A}) \in V$ for all $\mathscr{A} \in V$. Next, we will show that $\sigma \in P(V)$. Suppose that $\sigma \notin P(V)$. Then there is an identity $s \approx t \in \operatorname{Id}^{s} V$ such that $\hat{\sigma}[s] \approx \hat{\sigma}[t] \notin \operatorname{Id}^{s} V$ and this implies that there exists $\mathscr{A} \in V$ such that $\hat{\sigma}[s] \approx \hat{\sigma}[t] \notin \operatorname{Id}^{s} \mathscr{A}$. By Proposition 2.10, we get $s \approx t \notin \operatorname{Id}^{s} \sigma(\mathscr{A})$ and 
$\sigma(\mathscr{A}) \notin V$ which is a contradiction. So, $\sigma \in P(V)=\left[\sigma_{\text {id }}\right]_{\sim_{V-\text { iso }}}$ and $\sigma \sim_{V \text {-iso }} \sigma_{\text {id }}$. Therefore $\sigma(\mathscr{A}) \cong \mathscr{A}$ for all $\mathscr{A} \in V$. Then $V$ is fluid.

Let $V$ be a fluid strong variety of partial algebras of type $\tau$ and assume $W$ is a subvariety of $V$. Clearly, $W$ is also fluid since, for all $\mathscr{A} \in W \subseteq V$ and $\sigma \in \operatorname{Hyp}_{R}^{C}(\tau)$, we have

$$
\sigma(\mathscr{A}) \in W \Longrightarrow \sigma(\mathscr{A}) \cong \mathscr{A}
$$

Therefore, we have the following.

Proposition 4.9. Every subvariety of a fluid strong variety of partial algebras of type $\tau$ is fluid.

Definition 4.10. A strong variety $V$ of partial algebras of type $\tau$ is strongly fluid if, for every partial algebra $\mathscr{A} \in V$ and every hypersubstitution $\sigma \in \operatorname{Hyp}_{R}^{C}(\tau)$, there holds

$$
\sigma(\mathscr{A}) \in V \Longrightarrow \sigma(\mathscr{A})=\mathscr{A}
$$

Remark 4.11. If $V$ is strongly fluid, then $V$ is fluid.

Proposition 4.12. Let $V$ be a strong variety of partial algebras of type $\tau$.

(i) If $V$ is strongly fluid, then for all $\mathscr{A} \in V$, and for all $\sigma \in P(V), \sigma(\mathscr{A})=\mathscr{A}$.

(ii) If $V$ is strongly fluid, then $V$ is unsolid.

Proof. (i) Assume that $V$ is strongly fluid. Let $\mathscr{A} \in V$ and $\sigma \in P(V)$. By Proposition 4.6, we get $\sigma(\mathscr{A}) \in \sigma(V) \subseteq V$. Since $V$ is strongly fluid, we get $\sigma(\mathscr{A})=\mathscr{A}$.

(ii) Assume that $V$ is strongly fluid. By (i), for all $\mathscr{A} \in V$ and for all $\sigma \in P(V)$ we have $\sigma(\mathscr{A})=\mathscr{A}=\sigma_{\text {id }}(\mathscr{A})$ (i.e., $\sigma \sim_{V} \sigma_{\text {id }}$ and $\sigma\left(f_{i}\right) \approx f_{i}\left(x_{1}, \ldots, x_{n_{i}}\right) \in \operatorname{Id}^{s} V$ for all $i \in I$ ). This shows that $P(V) \subseteq P_{0}(V)$. But $P_{0}(V) \subseteq P(V)$ and then $P(V)=P_{0}(V)$. So, $V$ is unsolid.

Proposition 4.13. If $V$ is a fluid strong variety of partial algebras of type $\tau$ and $\left[\sigma_{\mathrm{id}}\right]_{\sim_{V}}=$ $\left[\sigma_{\text {id }}\right]_{\sim_{V-\text { iso }}}$, then $V$ is unsolid.

Proof. Assume that $V$ is fluid and $\left[\sigma_{\mathrm{id}}\right]_{\sim_{V}}=\left[\sigma_{\mathrm{id}}\right]_{\sim_{V-\text { iso }}}$. Let $\sigma \in P(V)$. Since $V$ is fluid, we get $\sigma(\mathscr{A}) \cong \mathscr{A}$ for all $\mathscr{A} \in V$ (i.e., $\left.\sigma \sim_{V \text {-iso }} \sigma_{\text {id }}\right)$. Therefore $\sigma \in\left[\sigma_{\text {id }}\right]_{\sim_{V-\text { iso }}}=\left[\sigma_{\text {id }}\right]_{\sim_{V}}$ (i.e., $\left.\sigma \sim_{V} \sigma_{\text {id }}\right)$ and we have $\sigma \in P_{0}(V)$. So $P(V) \subseteq P_{0}(V)$, but since $P_{0}(V) \subseteq P(V)$, then $P(V)=P_{0}(V)$. Therefore $V$ is unsolid.

Proposition 4.14. Let $V$ be a strong variety of partial algebras of type $\tau$. Then $\left.\sim_{V}\right|_{P(V)}$ is a congruence relation on the algebra $\left(P(V) ;{ }^{\circ}, \sigma_{\mathrm{id}}\right)$.

Proof. Let $\sigma_{1}, \sigma_{2} \in P(V)$ such that $\left.\sigma_{1} \sim_{V}\right|_{P(V)} \sigma_{2}$ and let $\sigma \in P(V)$. Then $\sigma(\mathscr{A}) \in V$ for all $\mathscr{A} \in V$.

We show that $\left.\sim_{V}\right|_{P(V)}$ is a right congruence.

$\left.\sigma_{1} \sim_{V}\right|_{P(V)} \sigma_{2}$ implies that $\sigma_{1}(\mathscr{A})=\sigma_{2}(\mathscr{A})$ for all $\mathscr{A} \in V$ and we get that $\sigma\left(\sigma_{1}(\mathscr{A})\right)=$ $\sigma\left(\sigma_{2}(\mathscr{A})\right)$ since $\sigma$ is a function. So, $\sigma_{1}{ }^{\circ}{ }_{h} \sigma \sim_{V} \sigma_{2}{ }^{\circ}{ }_{h} \sigma$ but $\sigma_{1}{ }^{\circ}{ }_{h} \sigma, \sigma_{2}{ }^{\circ} h \in \in P(V)$ because $P(V)$ is a monoid. Therefore $\left.\sigma_{1}{ }^{\circ}{ }_{h} \sigma \sim_{V}\right|_{P(V)} \sigma_{2}{ }^{\circ}{ }_{h} \sigma$.

We show that $\left.\sim_{V}\right|_{P(V)}$ is a left congruence. 
$\sigma(\mathscr{A}) \in V$ and $\left.\sigma_{1} \sim_{V}\right|_{P(V)} \sigma_{2}$ imply that $\sigma_{1}(\sigma(\mathscr{A}))=\sigma_{2}(\sigma(\mathscr{A}))$. So, $\sigma \circ_{h} \sigma_{1} \sim_{V} \sigma \circ_{h} \sigma_{2}$ but $\sigma \circ_{h} \sigma_{1}, \sigma \circ_{h} \sigma_{2} \in P(V)$ because $P(V)$ is a monoid. Therefore $\left.\sigma \circ_{h} \sigma_{1} \sim_{V}\right|_{P(V)} \sigma \circ_{h} \sigma_{2}$.

So, $\left.\sim_{V}\right|_{P(V)}$ is a congruence relation.

Proposition 4.15. Let $V$ be a strong variety of partial algebras of type $\tau$. Then $\left.\sim_{V-\text { iso }}\right|_{P(V)}$ is a congruence relation on the algebra $\left(P(V) ;{ }^{\circ} h, \sigma_{\mathrm{id}}\right)$.

Proof. Let $\sigma_{1}, \sigma_{2} \in P(V)$ such that $\left.\sigma_{1} \sim_{V \text {-iso }}\right|_{P(V)} \sigma_{2}$ and let $\sigma \in P(V)$. Then $\sigma(\mathscr{A}) \in V$ for all $\mathscr{A} \in V$.

We show that $\sim_{V-\text { iso }} \mid P(V)$ is a right congruence.

$\left.\sigma_{1} \sim_{V \text {-iso }}\right|_{P(V)} \sigma_{2}$ implies that $\sigma_{1}(\mathscr{A}) \cong \sigma_{2}(\mathscr{A})$ for all $\mathscr{A} \in V$ and by Lemma 3.13, we get that $\sigma\left(\sigma_{1}(\mathscr{A})\right) \cong \sigma\left(\sigma_{2}(\mathscr{A})\right)$. So, $\sigma_{1}{ }^{\circ}{ }_{h} \sigma \sim_{V-\text { iso }} \sigma_{2}{ }^{\circ}{ }_{h} \sigma$ but $\sigma_{1}{ }^{\circ}{ }_{h} \sigma, \sigma_{2}{ }^{\circ}{ }_{h} \sigma \in P(V)$ because $P(V)$ is a monoid. Therefore $\left.\sigma_{1}{ }^{\circ}{ }_{h} \sigma \sim_{V-\text { iso }}\right|_{P(V)} \sigma_{2}{ }^{\circ}{ }_{h} \sigma$.

We show that $\left.\sim_{V-\text { iso }}\right|_{P(V)}$ is a left congruence.

Since $\sigma(\mathscr{A}) \cong \sigma(\mathscr{A})$ and $\sigma(\mathscr{A}) \in V$, then $\sigma_{1}(\sigma(\mathscr{A})) \cong \sigma_{2}(\sigma(\mathscr{A}))$. So, $\sigma \circ_{h} \sigma_{1} \sim_{V-\text { iso }} \sigma \circ_{h}$ $\sigma_{2}$ but $\sigma{ }^{\circ} \sigma_{1}, \sigma \circ_{h} \sigma_{2} \in P(V)$ because $P(V)$ is a monoid. Therefore $\left.\sigma \circ_{h} \sigma_{1} \sim_{V \text {-iso }}\right|_{P(V)} \sigma \circ_{h}$ $\sigma_{2}$.

So, $\left.\sim_{V-\text { iso }}\right|_{P(V)}$ is a congruence relation.

\section{5. $n$-fluid and $n$-unsolid strong varieties}

The concepts of fluid and unsolid strong varieties of partial algebras can be generalized in the following way.

Definition 5.1. Let $1 \leq n \in \mathbb{N}$. A strong variety $V$ of partial algebras of type $\tau$ is called $n$-fluid, if there are $\sigma_{1}, \ldots, \sigma_{n} \in P(V)$ with $\sigma_{i} \chi_{V \text {-iso }} \sigma_{j}$ for $1 \leq i \neq j \leq n$ such that for all $\mathscr{A} \in V$ and for all $\sigma \in \operatorname{Hyp}_{R}^{C}(\tau)$ the following implication holds:

$(*)$ if $\sigma(\mathscr{A}) \in V$, then there is a $k \in\{1, \ldots, n\}$ with $\sigma(\mathscr{A}) \cong \sigma_{k}(\mathscr{A})$.

Proposition 5.2. Let $V$ be an $n$-fluid strong variety of partial algebras of type $\tau$. Then $\left|P(V) / \sim_{V-\text { iso }}\right|_{P(V)} \mid \geq n$.

Proof. Since $V$ is $n$-fluid, there are $\sigma_{1}, \ldots, \sigma_{n} \in P(V)$ with $\sigma_{i} \chi_{V \text {-iso }} \sigma_{j}$ for all $1 \leq i \neq j \leq n$ such that condition $(*)$ is satisfied. Since $\left[\sigma_{i}\right]_{\left.\sim_{V-\text { iso }}\right|_{P(V)}} \subseteq P(V)$ for all $i \in\{1, \ldots, n\}$ we have $\left[\sigma_{1}\right]_{\left.\sim_{V-\text { iso }}\right|_{P(V)}} \cup \cdots \cup\left[\sigma_{n}\right]_{\sim_{V \text {-iso } \mid P(V)}} \subseteq P(V)$ and $\left|P(V) / \sim_{V \text {-iso }}\right| P(V) \mid \geq n$.

Definition 5.3. A strong variety $V$ of partial algebras of type $\tau$ is called $n$-unsolid if and only if $\left|P(V) / \sim_{V}\right|_{P(V)} \mid=n$.

By this definition, we have that if $V$ is $n$-unsolid, then $P(V)=\left[\sigma_{1}\right]_{\left.\sim_{V}\right|_{P(V)}} \cup \cdots \cup$ $\left.\sim_{V}\right|_{P(V)}$. Where $\sigma_{i} \chi_{V} \sigma_{j}$ for all $1 \leq i \neq j \leq n$. But $\left[\sigma_{i}\right]_{\left.\sim_{V}\right|_{P(V)}} \subseteq\left[\sigma_{i}\right]_{\left.\sim_{V-\text { iso }}\right|_{P(V)}} \subseteq P(V)$ for all $i \in\{1, \ldots, n\}$. So $P(V)=\left[\sigma_{1}\right]_{\sim_{V-\text { iso }} \mid P(V)} \cup \cdots \cup\left[\sigma_{n}\right]_{\sim_{V-\text { iso }} \mid P(V)}$. We have that if $V$ is $n$ unsolid, then $P(V)=\left[\sigma_{1}\right]_{\left.\sim_{V}\right|_{P(V)}} \cup \cdots \cup\left[\sigma_{n}\right]_{\left.\sim_{V}\right|_{P(V)}}=\left[\sigma_{1}\right]_{\left.\sim_{V-\text { iso }}\right|_{P(V)}} \cup \cdots \cup\left[\sigma_{n}\right]_{\left.\sim_{V-\text { iso }}\right|_{P(V)}}$.

The following concept generalizes that of an $n$-fluid variety.

Proposition 5.4. Let $1 \leq n \in \mathbb{N}$ and let $V$ be a strong variety of partial algebras of type $\tau$ with $\left.\sim_{V}\right|_{P(V)}=\left.\sim_{V \text {-iso }}\right|_{P(V)}$. If $V$ is n-fluid, then $V$ is $k$-unsolid for $k \geq n$. 
Proof. Assume that $V$ is $n$-fluid. Then we have $\left|P(V) / \sim_{V \text {-iso }}\right|_{P(V)} \mid \geq n$. Since $\sim_{V} \mid P(V)=$ $\sim_{V \text {-iso }} \mid P(V)$ we get $\left|P(V) / \sim_{V \text {-iso }\left.\right|_{P(V)}}\right|=\left|P(V) / \sim_{V \mid P(V)}\right|=k$, that is, $V$ is $k$-unsolid.

\section{Examples}

Let $B$ be the strong regular variety

$$
B=\operatorname{Mod}^{s}\left\{x_{1}\left(x_{2} x_{3}\right) \approx\left(x_{1} x_{2}\right) x_{3}, x_{1}^{2} \approx x_{1}\right\}
$$

that is, the class of all partial algebras of type (2) which satisfy the associative and the idempotent law as strong identities. Both equations are regular (i.e., the both sides of the equation have the same variables occurring). We denote by $\sigma_{t} \in \operatorname{Hyp}_{R}^{C}(2)$ the regular hypersubstitution which maps the binary operation symbol $f$ to the term $t \in W_{(2)}^{C}\left(\left\{x_{1}, x_{2}\right\}\right)$. Instead of $f\left(x_{1}, x_{2}\right)$ we write simply $x_{1} x_{2}$. The set $\operatorname{Hyp}_{R}^{C}(2) / \sim_{B}$ consists precisely of the following classes of hypersubstitutions: $\left[\sigma_{\varepsilon_{1}^{2}\left(x_{1}, x_{2}\right)}\right]_{\sim_{B}},\left[\sigma_{\varepsilon_{2}^{2}\left(x_{1}, x_{2}\right)}\right]_{\sim_{B}},\left[\sigma_{x_{1} x_{2}}\right]_{\sim_{B}},\left[\sigma_{x_{2} x_{1}}\right]_{\sim_{B}}$, $\sim_{B},\left[\sigma_{x_{2} x_{1} x_{2}}\right]_{\sim_{B}}$. We will be particularly interested in the following strong regular subvarieties of the strong regular variety $B$ :

$$
\begin{gathered}
\mathrm{TR}=\operatorname{Mod}^{s}\left\{\varepsilon_{1}^{2}\left(x_{1}, x_{2}\right) \approx \varepsilon_{2}^{2}\left(x_{1}, x_{2}\right)\right\}, \\
\mathrm{LZ}=\operatorname{Mod}^{s}\left\{x_{1} x_{2} \approx \varepsilon_{1}^{2}\left(x_{1}, x_{2}\right)\right\}, \\
\mathrm{RZ}=\operatorname{Mod}^{s}\left\{x_{1} x_{2} \approx \varepsilon_{2}^{2}\left(x_{1}, x_{2}\right)\right\}, \\
\mathrm{SL}=\operatorname{Mod}^{s}\left\{x_{1}\left(x_{2} x_{3}\right) \approx\left(x_{1} x_{2}\right) x_{3}, x_{1}^{2} \approx x_{1}, x_{1} x_{2} \approx x_{2} x_{1}\right\}, \\
\mathrm{RB}=\operatorname{Mod}^{s}\left\{x_{1}\left(x_{2} x_{3}\right) \approx\left(x_{1} x_{2}\right) x_{3} \approx \varepsilon_{1}^{2}\left(x_{1}, x_{2}\right) x_{3}, x_{1}^{2} \approx x_{1}\right\}, \\
\mathrm{NB}=\operatorname{Mod}^{s}\left\{x_{1}\left(x_{2} x_{3}\right) \approx\left(x_{1} x_{2}\right) x_{3}, x_{1}^{2} \approx x_{1}, x_{1} x_{2} x_{3} x_{4} \approx x_{1} x_{3} x_{2} x_{4}\right\}, \\
\mathrm{RegB}=\operatorname{Mod}^{s}\left\{x_{1}\left(x_{2} x_{3}\right) \approx\left(x_{1} x_{2}\right) x_{3}, x_{1}^{2} \approx x_{1}, x_{1} x_{2} x_{1} x_{3} x_{1} \approx x_{1} x_{2} x_{3} x_{1}\right\}, \\
\mathrm{LN}=\operatorname{Mod}^{s}\left\{x_{1}\left(x_{2} x_{3}\right) \approx\left(x_{1} x_{2}\right) x_{3}, x_{1}^{2} \approx x_{1}, x_{1} x_{2} x_{3} \approx x_{1} x_{3} x_{2}\right\}, \\
\mathrm{RN}=\operatorname{Mod}^{s}\left\{x_{1}\left(x_{2} x_{3}\right) \approx\left(x_{1} x_{2}\right) x_{3}, x_{1}^{2} \approx x_{1}, x_{1} x_{2} x_{3} \approx x_{2} x_{1} x_{3}\right\}, \\
\mathrm{LReg}=\operatorname{Mod}^{s}\left\{x_{1}\left(x_{2} x_{3}\right) \approx\left(x_{1} x_{2}\right) x_{3}, x_{1}^{2} \approx x_{1}, x_{1} x_{2} \approx x_{1} x_{2} x_{1}\right\}, \\
\mathrm{RReg}=\operatorname{Mod}^{s}\left\{x_{1}\left(x_{2} x_{3}\right) \approx\left(x_{1} x_{2}\right) x_{3}, x_{1}^{2} \approx x_{1}, x_{1} x_{2} \approx x_{2} x_{1} x_{2}\right\}, \\
\mathrm{LQN}=\operatorname{Mod}^{s}\left\{x_{1}\left(x_{2} x_{3}\right) \approx\left(x_{1} x_{2}\right) x_{3}, x_{1}^{2} \approx x_{1}, x_{1} x_{2} x_{3} \approx x_{1} x_{2} x_{1} x_{3}\right\}, \\
\mathrm{RQN}=\operatorname{Mod}^{s}\left\{x_{1}\left(x_{2} x_{3}\right) \approx\left(x_{1} x_{2}\right) x_{3}, x_{1}^{2} \approx x_{1}, x_{1} x_{2} x_{3} \approx x_{1} x_{3} x_{2} x_{3}\right\} .
\end{gathered}
$$

All these varieties are strong regular varieties of partial algebras.

These varieties are given in Figure 6.1.

This is not the lattice of all strong subvarieties of $B$ since we consider strong regular ones.

A strong variety $V$ of partial algebras of type (2) is called dual solid if from $s \approx t \in \operatorname{Id}^{s} V$ there follows $\hat{\sigma}_{x_{2} x_{1}}[s] \approx \hat{\sigma}_{x_{2} x_{1}}[t] \in \operatorname{Id}^{s} V$. Then we have the following results. 


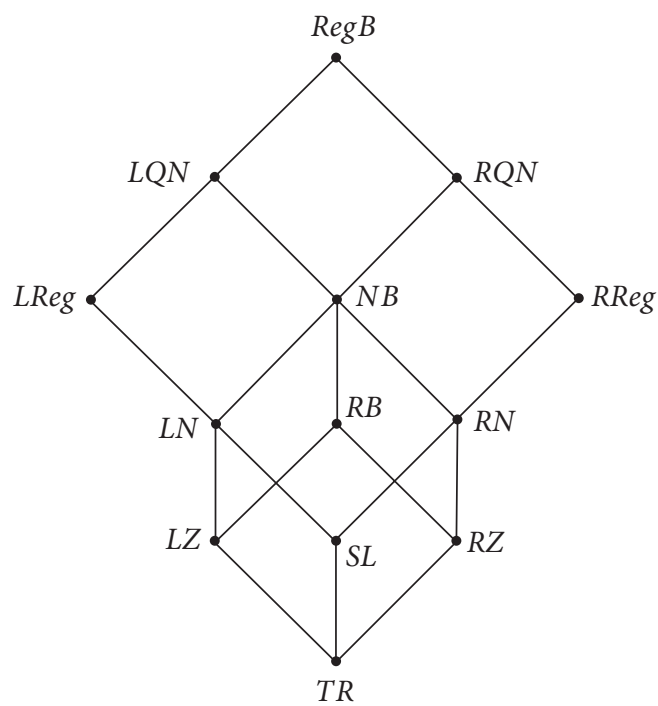

Figure 6.1

Theorem 6.1. (1) TR, LZ, RZ, SL are unsolid.

(2) LN, RN, LReg, RReg are 2-unsolid.

(3) B, RB, LQN, RQN are 4-unsolid.

(4) NB and RegB are 6-unsolid.

(5) All dual solid varieties different from TR, SL, NB, and RegB are 4-unsolid.

(6) Any variety other than LZ, RZ, LN, RN, LReg, RReg, LQN, RQN which is not dualsolid is 3-unsolid.

Proof. (1) It is easy to see that TR, LZ, RZ are unsolid. Further, $\operatorname{Hyp}_{R}^{C}(2)=\left[\sigma_{\varepsilon_{1}^{2}\left(x_{1}, x_{2}\right)}\right]_{\sim_{S L}} U$ $\left[\sigma_{\varepsilon_{2}^{2}\left(x_{1}, x_{2}\right)}\right]_{\sim_{\mathrm{SL}}} \cup\left[\sigma_{x_{1} x_{2}}\right]_{\sim_{\mathrm{SL}}}$, where $\sigma_{x_{1} x_{2}} \in P(\mathrm{SL})$. The application of $\sigma_{\varepsilon_{1}^{2}\left(x_{1}, x_{2}\right)}$ to $x_{1} x_{2} \approx x_{2} x_{1} \in$ $\mathrm{Id}^{s} \mathrm{SL}$ provides $x_{1} \approx x_{2} \notin \mathrm{Id}^{s} \mathrm{SL}$ and the application of $\sigma_{\varepsilon_{2}^{2}\left(x_{1}, x_{2}\right)}$ to $x_{1} x_{2} \approx x_{2} x_{1}$ provides $x_{2} \approx x_{1} \notin \mathrm{Id}^{s} \mathrm{SL}$. This shows that $\sigma_{\varepsilon_{1}^{2}\left(x_{1}, x_{2}\right)}, \sigma_{\varepsilon_{2}^{2}\left(x_{1}, x_{2}\right)} \notin P(\mathrm{SL})$. Consequently, $|P(\mathrm{SL})|=$ $\left|\left[\sigma_{x_{1} x_{2}}\right]_{\sim_{\mathrm{SL}}}\right|=1$, that is, SL is unsolid.

(2) It is easy to see that $\operatorname{Hyp}_{R}^{C}(2)=\left[\sigma_{\varepsilon_{1}^{2}\left(x_{1}, x_{2}\right)}\right]_{\sim_{\mathrm{LN}}} \cup\left[\sigma_{\varepsilon_{2}^{2}\left(x_{1}, x_{2}\right)}\right]_{\sim_{\mathrm{LN}}} \cup\left[\sigma_{x_{1} x_{2}}\right]_{\sim_{\mathrm{LN}}} \cup$ $\sim_{\mathrm{LN}}$, where $\sigma_{\varepsilon_{1}^{2}\left(x_{1}, x_{2}\right)}, \sigma_{x_{1} x_{2}} \in P(\mathrm{LN})$. If we apply $\hat{\sigma}_{\varepsilon_{2}^{2}\left(x_{1}, x_{2}\right)}$ to $x_{1} x_{2} x_{3} \approx x_{1} x_{3} x_{2}$, we obtain $x_{3} \approx$ $x_{2}$ which is not satisfied in LN and applying $\hat{\sigma}_{x_{2} x_{1}}$ to $x_{1} x_{2} x_{3} \approx x_{1} x_{3} x_{2}$ gives $x_{3} x_{2} x_{1} \approx x_{2} x_{3} x_{1}$ which is also not satisfied. Therefore $P(\mathrm{LN}) / \sim_{\mathrm{LN} \mid \mathrm{P}(\mathrm{LN})}=\left\{\left[\sigma_{\varepsilon_{1}^{2}\left(x_{1}, x_{2}\right)}\right]_{\sim_{\mathrm{LN}}},\left[\sigma_{x_{1} x_{2}}\right]_{\sim_{\mathrm{LN}}}\right\}$, that is, $\mathrm{LN}$ is 2-unsolid. Similarly we can show that RN is 2-unsolid. For LReg and RReg we show in a similar way that these strong varieties are 2-unsolid.

(3) It is easy to check that $\operatorname{Hyp}_{R}^{C}(2)=\left[\sigma_{\varepsilon_{1}^{2}\left(x_{1}, x_{2}\right)}\right]_{\sim_{B}} \cup\left[\sigma_{\varepsilon_{2}^{2}\left(x_{1}, x_{2}\right)}\right]_{\sim_{B}} \cup\left[\sigma_{x_{1} x_{2}}\right]_{\sim_{B}} \cup\left[\sigma_{x_{2} x_{1}}\right]_{\sim_{B}} \cup$ $\left[\sigma_{x_{1} x_{2} x_{1}}\right]_{\sim_{B}} \cup\left[\sigma_{x_{2} x_{1} x_{2}}\right]_{\sim_{B}}$, where $\sigma_{\varepsilon_{1}^{2}\left(x_{1}, x_{2}\right)}, \sigma_{\varepsilon_{2}^{2}\left(x_{1}, x_{2}\right)}, \sigma_{x_{1} x_{2}}, \sigma_{x_{2} x_{1}} \in P(B)$. The application of $\sigma_{x_{1} x_{2} x_{1}}$ to the associative law provides $x_{1} x_{2} x_{1} x_{3} x_{1} x_{2} x_{1} \approx x_{1} x_{2} x_{3} x_{2} x_{1} \notin \mathrm{Id}^{s} B$ and the application of $\sigma_{x_{2} x_{1} x_{2}}$ to the associative law provides $x_{3} x_{2} x_{1} x_{2} x_{3} \approx x_{3} x_{2} x_{3} x_{1} x_{3} x_{2} x_{3} \notin \operatorname{Id}^{s} B$. This shows that $\sigma_{x_{1} x_{2} x_{1}}, \sigma_{x_{2} x_{1} x_{2}} \notin P(B)$. Consequently, $\left|P(B) / \sim_{B \mid P(B)}\right|=\mid\left\{\left[\sigma_{\varepsilon_{1}^{2}\left(x_{1}, x_{2}\right)}\right]_{\sim_{B}},\left[\sigma_{\varepsilon_{2}^{2}\left(x_{1}, x_{2}\right)}\right]_{\sim_{B}}\right.$, $\left.\sim_{B},\left[\sigma_{x_{2} x_{1}}\right]_{\sim_{B}}\right\} \mid=4$, that is, $B$ is 4-unsolid. Further we have $\operatorname{Hyp}_{R}^{C}(2)=\left[\sigma_{\varepsilon_{1}^{2}\left(x_{1}, x_{2}\right)}\right]_{\sim_{\mathrm{RB}}} \cup$ $\left[\sigma_{\varepsilon_{2}^{2}\left(x_{1}, x_{2}\right)}\right]_{\sim_{\mathrm{RB}}} \cup\left[\sigma_{x_{1} x_{2}}\right]_{\sim_{\mathrm{RB}}} \cup\left[\sigma_{x_{2} x_{1}}\right]_{\sim_{\mathrm{RB}}}$, where $\sigma_{\varepsilon_{1}^{2}\left(x_{1}, x_{2}\right)}, \sigma_{\varepsilon_{2}^{2}\left(x_{1}, x_{2}\right)}, \sigma_{x_{1} x_{2}}, \sigma_{x_{2} x_{1}} \in P(\mathrm{RB})$ and 
$\left|P(\mathrm{RB}) /_{\sim_{B \mid P(\mathrm{RB})}}\right|=\left|\left\{\left[\sigma_{\varepsilon_{1}^{2}\left(x_{1}, x_{2}\right)}\right]_{\sim_{\mathrm{RB}}},\left[\sigma_{\varepsilon_{2}^{2}\left(x_{1}, x_{2}\right)}\right]_{\sim_{\mathrm{RB}}},\left[\sigma_{x_{1} x_{2}}\right]_{\sim_{\mathrm{RB}}},\left[\sigma_{x_{2} x_{1}}\right]_{\sim_{\mathrm{RB}}}\right\}\right|=4$, that is, RB is 4-unsolid. In a similar way one proves that LQN as well as RQN are 4-unsolid.

(4) It is easy to check that $\operatorname{Hyp}_{R}^{C}(2)=\left[\sigma_{\varepsilon_{1}^{2}\left(x_{1}, x_{2}\right)}\right]_{\sim_{\mathrm{NB}}} \cup\left[\sigma_{\varepsilon_{2}^{2}\left(x_{1}, x_{2}\right)}\right]_{\sim_{\mathrm{NB}}} \cup\left[\sigma_{x_{1} x_{2}}\right]_{\sim_{\mathrm{NB}}} \cup$ $\sim_{\mathrm{NB}} \cup\left[\sigma_{x_{1} x_{2} x_{1}}\right]_{\sim_{\mathrm{NB}}} \cup\left[\sigma_{x_{2} x_{1} x_{2}}\right]_{\sim_{\mathrm{NB}}}$. All these hypersubstitutions are NB-proper, that is, NB is solid. This gives $\left|P(\mathrm{NB}) / \sim_{\mathrm{NB}}\right|=6$, that is, NB is 6-unsolid. In a similar way one proves that RegB is 6-unsolid.

(5) Let now $V$ be a dual solid variety different from TR, SL, RB, NB, and RegB. Then we have $\operatorname{Hyp}_{R}^{C}(2)=\left[\sigma_{\varepsilon_{1}^{2}\left(x_{1}, x_{2}\right)}\right]_{\sim_{V}} \cup\left[\sigma_{\varepsilon_{2}^{2}\left(x_{1}, x_{2}\right)}\right]_{\sim_{V}} \cup\left[\sigma_{x_{1} x_{2}}\right]_{\sim_{V}} \cup\left[\sigma_{x_{2} x_{1}}\right]_{\sim_{V}} \cup\left[\sigma_{x_{1} x_{2} x_{1}}\right]_{\sim_{V}} \cup$ $\sim_{V}$. Since $V$ is dual solid, the hypersubstitutions $\sigma_{x_{1} x_{2}}$ and $\sigma_{x_{2} x_{1}}$ are $V$-proper. As a consequence of $V \neq \mathrm{TR}, \mathrm{SL}$ and since $V$ is dual solid we have $\sigma_{\varepsilon_{1}^{2}\left(x_{1}, x_{2}\right)}, \sigma_{\varepsilon_{2}^{2}\left(x_{1}, x_{2}\right)} \in P(V)$. The application of $\sigma_{x_{1} x_{2} x_{1}}$ to the associative law provides $x_{1} x_{2} x_{3} x_{2} x_{1} \approx x_{1} x_{2} x_{1} x_{3} x_{1} x_{2} x_{1}$. From this equation we derive $x_{1} x_{2} x_{3} x_{1} \approx x_{1} x_{2} x_{1} x_{3} x_{1}$ in the following way:

$$
\begin{aligned}
x_{1} x_{2} x_{3} x_{1} & \approx x_{1} x_{2} x_{3} x_{3} x_{2} x_{3} x_{1} \\
& \approx x_{1} x_{2} x_{3} x_{1} x_{3} x_{1} x_{2} x_{3} x_{1} \\
& \approx x_{1} x_{2} x_{3} x_{1} x_{3} x_{1} x_{2} x_{1} x_{3} x_{1} \\
& \approx x_{1} x_{2} x_{1} x_{3} x_{1} x_{3} x_{1} x_{2} x_{1} x_{3} x_{1} \\
& \approx x_{1} x_{2} x_{1} x_{3} x_{1} x_{2} x_{1} x_{3} x_{1} \\
& \approx x_{1} x_{2} x_{1} x_{3} x_{1} x_{1} x_{2} x_{1} x_{3} x_{1} \\
& \approx x_{1} x_{2} x_{1} x_{3} x_{1}
\end{aligned}
$$

This shows $V \subseteq$ RegB. But TR, SL, RB, NB, and RegB are the only dual solid subvarieties of RegB. Since $V$ is different from these varieties, we have $\sigma_{x_{1} x_{2} x_{1}} \notin P(V)$. The same argument shows $\sigma_{x_{2} x_{1} x_{2}} \notin P(V)$. Since $\mathrm{RB} \subseteq V$, the set $\operatorname{Id}^{s} V$ of all strong identities satisfied in $V$ consists only of outermost identities and this shows $\left|P(V) / \sim_{V \mid P(V)}\right|=$ $\left|\left\{\left[\sigma_{\varepsilon_{1}^{2}\left(x_{1}, x_{2}\right)}\right]_{\sim_{V}},\left[\sigma_{\varepsilon_{2}^{2}\left(x_{1}, x_{2}\right)}\right]_{\sim_{V}},\left[\sigma_{x_{1} x_{2}}\right]_{\sim_{V}},\left[\sigma_{x_{2} x_{1}}\right]_{\sim_{V}}\right\}\right|=4$, that is, $V$ is 4-unsolid.

(6) Finally if $V$ is not a dual solid variety different from LZ, RZ, LN, RN, LReg, RReg, LQN, RQN, then $\operatorname{Hyp}_{R}^{C}(2)=\left[\sigma_{\varepsilon_{1}^{2}\left(x_{1}, x_{2}\right)}\right]_{\sim_{V}} \cup\left[\sigma_{\varepsilon_{2}^{2}\left(x_{1}, x_{2}\right)}\right]_{\sim_{V}} \cup\left[\sigma_{x_{1} x_{2}}\right]_{\sim_{V}} \cup\left[\sigma_{x_{2} x_{1}}\right]_{\sim_{V}} \cup$ $\left[\sigma_{x_{1} x_{2} x_{1}}\right]_{\sim_{V}} \cup\left[\sigma_{x_{2} x_{1} x_{2}}\right]_{\sim_{V}}$. We can prove that $\sigma_{x_{2} x_{1}}, \sigma_{x_{1} x_{2} x_{1}}, \sigma_{x_{2} x_{1} x_{2}} \notin P(V)$. Then $\mid P(V) /$ $\sim_{V \mid P(V)}|=|\left\{\left[\sigma_{\varepsilon_{1}^{2}\left(x_{1}, x_{2}\right)}\right]_{\sim_{V}},\left[\sigma_{\varepsilon_{2}^{2}\left(x_{1}, x_{2}\right)}\right]_{\sim_{V}},\left[\sigma_{x_{1} x_{2}}\right]_{\sim_{V}}\right\} \mid=3$, that is, $V$ is 3 -unsolid.

\section{References}

[1] F. Börner, Varieties of partial algebras, Beiträge zur Algebra und Geometrie 37 (1996), no. 2, 259-287.

[2] P. Burmeister, A Model Theoretic Oriented Approach to Partial Algebras, Mathematical Research, vol. 32, Akademie, Berlin, 1986.

[3] S. Busaman and K. Denecke, Strong regular n-full varieties of partial algebras, Southeast Asian Bulletin of Mathematics 29 (2005), no. 2, 259-276.

[4] W. Craig, Near-equational and equational systems of logic for partial functions. I, The Journal of Symbolic Logic 54 (1989), no. 3, 795-827.

[5] _ Near-equational and equational systems of logic for partial functions. II, The Journal of Symbolic Logic 54 (1989), no. 4, 1181-1215.

[6] K. Denecke and S. L. Wismath, Hyperidentities and Clones, Algebra, Logic and Applications, vol. 14, Gordon and Breach Science, Amsterdam, 2000. 
[7] __ Universal Algebra and Applications in Theoretical Computer Science, Chapman \& Hall/CRC, Florida, 2002.

[8] H.-J. Hoehnke, Superposition partieller Funktionen, Studien zur Algebra und ihre Anwendungen, Schriftenreihe Zentralinst. Math. Mech. Akad. Wissensch. DDR, no. 16, Akademie, Berlin, 1972, pp. 7-26.

[9] J. Koppitz and K. Denecke, M-Solid Varieties of Algebras, Advances in Mathematics, vol. 10, Springer, New York, 2006.

[10] B. Staruch and B. Staruch, Strong regular varieties of partial algebras, Algebra Universalis 31 (1994), no. 2, 157-176.

[11] D. Welke, Hyperidentitäten partieller algebren, Ph.D. thesis, Universität Potsdam, Potsdam, 1996.

S. Busaman: Department of Mathematics and Computer Science, Prince of Songkla University, Pattani 94000, Thailand

E-mail address: bsaofee@bunga.pn.psu.ac.th

K. Denecke: Institute of Mathematics, University of Potsdam, Am Neuen Palais, Potsdam 14415, Germany

E-mail address: kdenecke@rz.uni-potsdam.de 


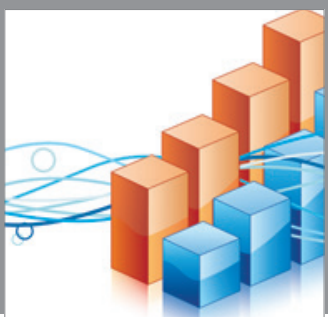

Advances in

Operations Research

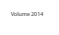

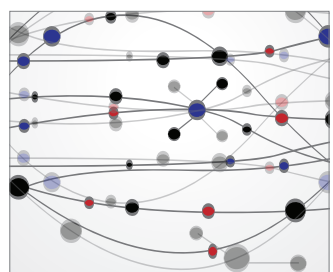

\section{The Scientific} World Journal
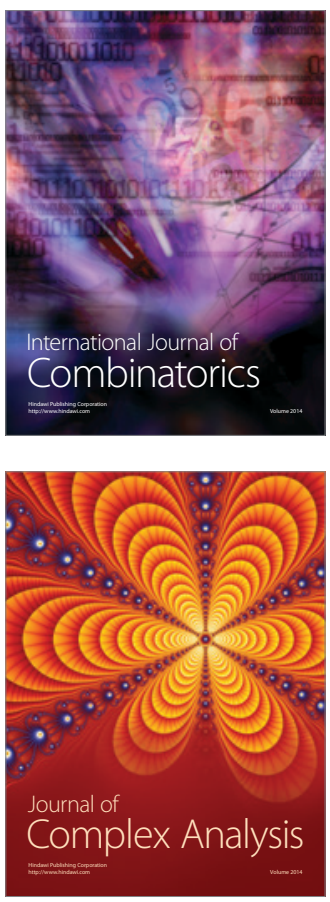

International Journal of

Mathematics and

Mathematical

Sciences
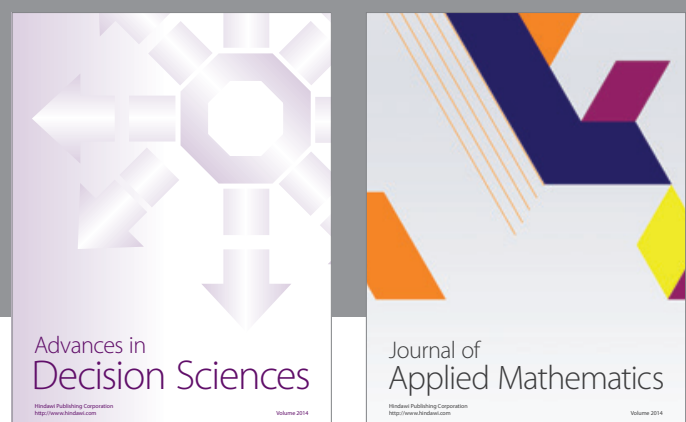

Journal of

Applied Mathematics
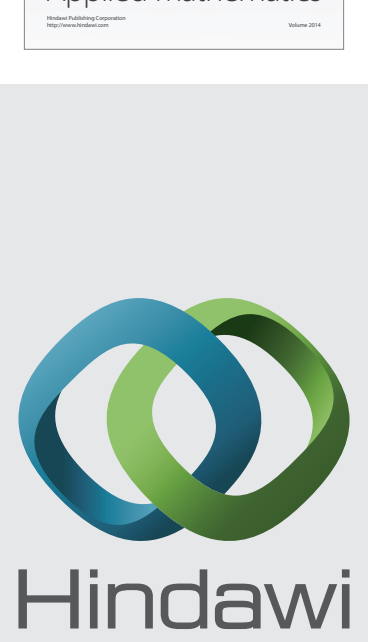

Submit your manuscripts at http://www.hindawi.com
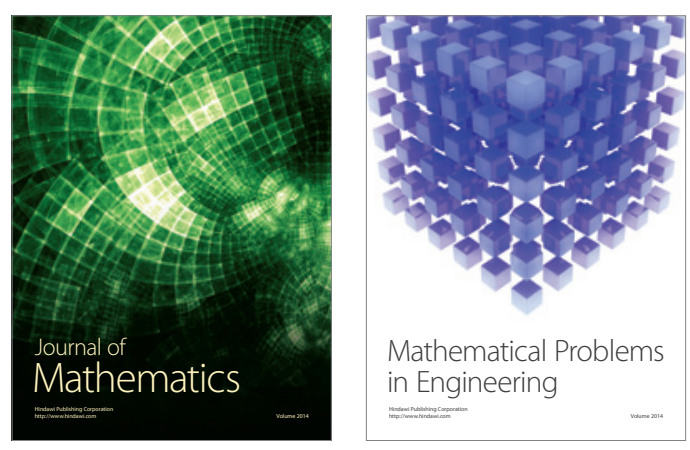

Mathematical Problems in Engineering
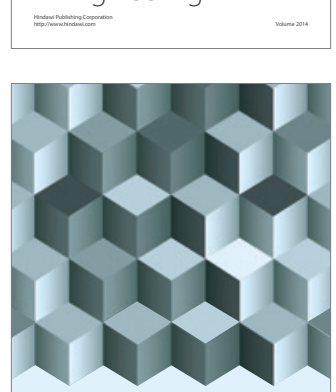

Journal of

Function Spaces
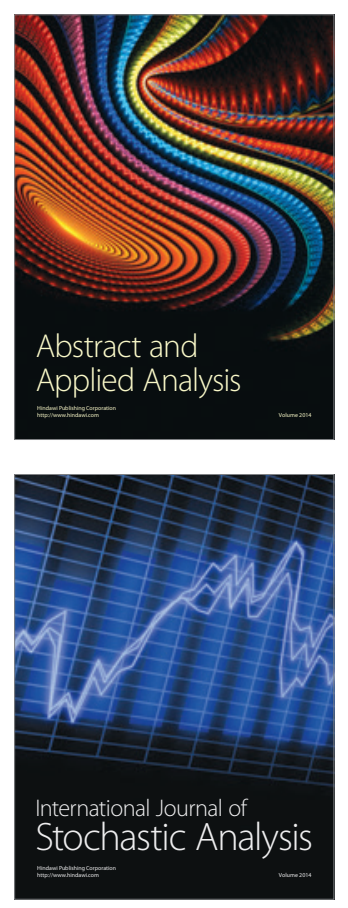

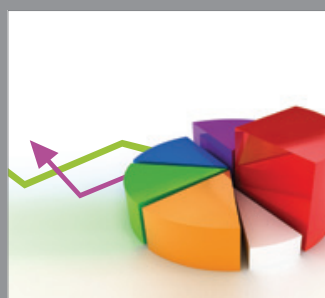

ournal of

Probability and Statistics

Promensencen
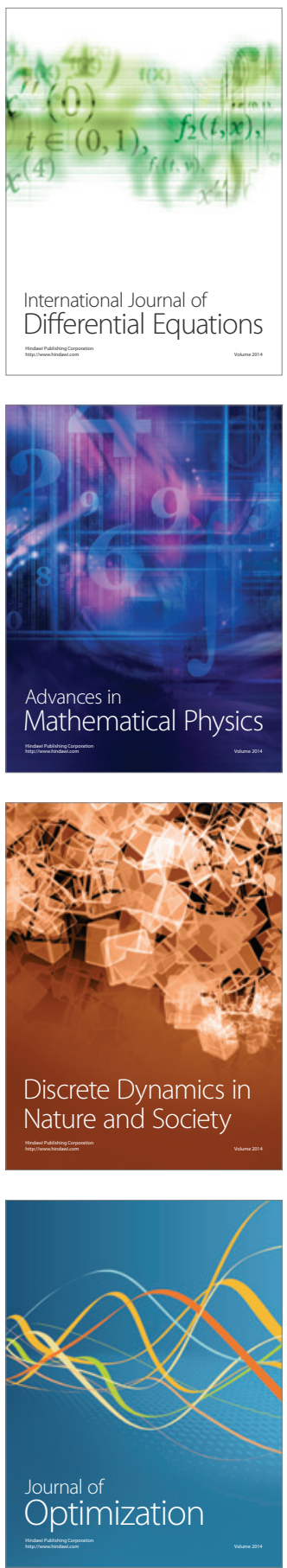